\title{
$\S 5$ Haftung des Account-Inhabers bei bewusster Weitergabe der Zugangsdaten
}

Durch die Weitergabe der Zugangsdaten wird ein Dritter willentlich in die Position gebracht, unter dem Namen des Account-Inhabers Willenserklärungen abzugeben 11 Diese willentliche Weitergabe begründet die Zurechnung eines etwaigen Rechtsscheins ${ }^{2}$ Dementsprechend existieren einige Lösungsansätze, den Account-Inhaber auf das positive Interesse des Geschäftspartners haften zu lassen, wenn er die Zugangsdaten weitergegeben hat. In der dogmatischen Begründung unterscheiden sich die Ansichten jedoch.

Beim Handeln über fremde Accounts im Internet finden die Stellvertretungsregeln wie beim Handeln unter fremdem Namen Anwendung 3 Bindung des Account-Inhabers an die Erklärung des Handelnden kommt somit in Betracht, wenn Vertretungsmacht bestand. Bei der Annahme die Übergabe sei eine konkludente Bevollmächtigung. ${ }^{4}$ fällt es schwer, den Umfang der Vollmacht zu bestimmen. Nur wenn der Dritte die Vollmacht im Außenverhältnis nicht überschreitet, jedoch im Innenverhältnis strengere Vorgaben hat, kann er die Vertretungsmacht so missbrauchen, dass eine Bindung des Vertretenen entsteht 5 Überschreitet der Dritte eine bestehende Vollmacht im Außenverhältnis kommt eine Bindung des Vertretenen nur mit dessen Zustimmung in Betracht (vgl. § 179 Abs. 1 BGB) $\rfloor^{6}$ In Fällen, in denen die Übergabe nicht als konkludente Bevollmächtigung zu sehen ist oder in denen der Dritte eine bestehende Vollmacht im Außenverhältnis

1 Dazu oben Rn. 125

2 Die willentliche Schaffung ist eine Fallgruppe der Zurechnung von Rechtsscheinen, dazu oben Rn. 249

3 Oben Rn. 283

4 In diese Richtung Spindler, in: Internet-Auktionen ${ }^{2}$, Kap. 5 Rn. 128, dagegen Herresthal, K\&R 2008, 705; ders., in: Taeger/Wiebe, 21, 26.

$5 \quad$ Faust, BGB AT ${ }^{3}, \S 28$ Rn. 23.

6 Siehe auch M. Wolf/Neuner ${ }^{10}$, 49 Rn. 100. 
überschreitet, stellt sich daher die Frage, ob der Account-Inhaber für den Missbrauch haften muss.

\section{Begriff der Weitergabe}

295 Entscheidende Vorfrage bei der Haftung für den Missbrauch von Zugangsdaten nach Weitergabe ist, was unter der Weitergabe verstanden wird. Bei engem Verständnis des Begriffs fallen nur Fälle darunter, bei denen der Account-Inhaber mit dem Bewusstsein, dass der Dritte die Zugangsdaten später eigenständig verwenden wird, ihm die Zugangsdaten mitteilt. $]^{7}$ Bei einem weiten Verständnis der Weitergabe fallen Konstellationen darunter, bei denen der Account-Inhaber dem Dritten die Zugangsdaten nicht wissentlich mitteilt 8 Unter Weitergabe fällt bei weitem Verständnis auch die Kenntnisnahme des Dritten durch das Lesen einer Notiz der Zugangsdater? das Speichern in der Schlüsselbund-Verwaltung ${ }^{10}$ des Rechners ${ }^{11}$ Unter die Weitergabe fällt nach keinem dieser Begriffe das Ausspähen der Zugangsdaten durch einen Phishing-Angriff: 12 Zwar gibt der Account-Inhaber die Zugangsdaten beim Phishing auch in ein Formular ein, er glaubt wegen der Täuschung jedoch sie dem Authentisierungsnehmer zur Authentisierung zu übermitteln, nicht einem Dritten zu offenbaren.

296 Bei dieser Untersuchung wird das enge Verständnis der Weitergabe zu Grunde gelegt. Bei der rechtlichen Bewertung macht es einen Unterschied, ob der Account-Inhaber dem Dritten im vollen Bewusstsein, dass dieser anschließend den Account verwenden kann und dies auch tun wird, die $\mathrm{Zu}$ gangsdaten mitgeteilt hat, oder ob der Dritte diese durch Nachlässigkeiten des Account-Inhabers erfährt. So zeigt beispielsweise der Blick auf $\S 172$ Abs. 1 BGB, dass eine solche Unterscheidung für die rechtliche Wertung von Bedeutung ist. Nach dem Wortlaut führt die willentliche Aushändi-

7 Für den engen Weitergabebegriff LG Bonn, Urteil v. 19. 12.2003, 2 O 472/03 MMR 2004, 179, 181; Oechsler, AcP 208 (2008), 565, 582.

8 Für ein weites Verständnis Borges, Elektronischer Identitätsnachweis, S. 136; ders., NJW 2011, 2400, 2403; Sonnentag, WM 2012, 1614, 1618; Verse/Gaschler, Jura 2009, 213, $215 \mathrm{f}$.

9 Dazu oben Rn. 132

10 Dazu oben Rn. $\overline{135}$

11 Borges, NJW 2011, 2400, 2403; Sonnentag, WM 2012, 1614, 1618.

12 Dazu Oben Rn. 138 
gung der Vollmachtsurkunde zu einer Zurechnung 13 Ob $§ 172$ Abs. 1 BGB analog auf den Fall des fahrlässigen Ermöglichens des Abhandenkommens der Vollmachtsurkunde übertragen werden kann, ist umstritten ${ }^{14}$ Unabhängig davon, ob beide Fälle im Ergebnis rechtlich gleich zu behandeln sind, zeigt die Unterscheidung, dass für beide Fälle andere Wertungen eine Rolle spielen. Um diese unterschiedlichen Wertungen berücksichtigen zu können, wird die Weitergabe hier eng verstanden.

\section{Lösung über die Duldungsvollmacht}

Ein Weg die Haftung des Account-Inhabers für den Missbrauch von $\mathrm{Zu}-$ gangsdaten nach deren Weitergabe zu lösen, besteht in der Anwendung der Grundsätze Rechtsscheinvollmacht in Form der Duldungsvollmacht ${ }^{15}$ Dieser Lösungsweg kann sowohl in Zwei- als auch in Drei-Personen-Konstellationen angewendet werden. Mit der Weitergabe der Zugangsdaten lasse es der Account-Inhaber wissentlich geschehen, dass der Dritte für ihn wie ein Vertreter auftrete und ein Geschäftsgegner nach Treu und Glauben dieses Verhalten als Bevollmächtigung verstehen könne ${ }^{16}$ Der Account-Inhaber hafte daher für den Rechtsschein in Form der Duldungsvollmacht ${ }^{17}$ In den Kategorien der Rechtsscheinhaftung getrennt bedeutet dies: Der Handelnde schafft durch die Verwendung des fremden Accounts einen Rechtsscheintatbestand. Diesen von einem Dritten geschaffenen Rechtsscheintatbestand muss sich der Handelnde zurechnen lassen, weil er ihn vorsätzlich durch die willentliche Weitergabe der Zugangsdaten hervorgerufen hat 18

13 Unten Rn. 314

14 Unten Rn. 315

15 Allgemein zur Duldungsvollmacht oben Rn.262

16 LG Aachen, Urteil v. 15. 12.2006, 5 S 184/06 - NJW-RR 2007, 565.

17 LG Aachen, Urteil v. 15. 12. 2006, 5 S 184/06 - NJW-RR 2007, 565; AG Saarbrücken, Urteil v. 15.2.2008, 37 C 1251/06, Rn. 31; Ellenberger, in: Palandt ${ }^{73}$, $\S 172$ BGB Rn. 18; Frensch, in: Prütting/Wegen/Weinreich ${ }^{8}$, § 167 BGB Rn. 41; Hanau, Handeln unter fremder Nummer, S. 40; Klein, MMR 2011, 450, 451; Kitz, in: Hoeren/Sieber/Holznagel, Kap. 13.1 Rn. 77; Mankowski, CR 2007, 606, 607; Schramm, in: MüKo-BGB ${ }^{6} \S 164$ Rn. 45a; Spindler/Anton, in: Spindler/F. Schus$t^{2}{ }^{2}, \S 164$ BGB Rn. 9. Wohl auch BGH, Urteil v. 11. 5. 2011, VIII ZR 289/09 (VIPBareinrichtung) - BGHZ 189, 346, Rn. 15; OLG Hamm, Urteil v. 16. 11.2006, 28 U 84/06 - NJW 2007, 611, 612. Haftung über die Anscheinsvollmacht: $O L G$ Schleswig, Beschluss v. 19. 7. 2010, 3 W 47/10 - CR 2011, 52.

Mankowski, CR 2007, 606, 607. 
298 Der Hinweis, dass der Account-Inhaber bereits mit der Weitergabe der Zugangsdaten gegen die AGB des Authentisierungsnehmers wie beispielsweise eine Internet-Auktionsplattform verstoßen hat ${ }^{19}$ kann - sofern der Authentisierungsnehmer im Einzelfall solche AGB einsetzt - nicht direkt zur Begründung der Duldungsvollmacht herangezogen werden. Eine Pflichtverletzung gegenüber dem Authentisierungsnehmer durch den Account-Inhaber wirkt sich nicht auf das Verhältnis zum insoweit unbeteiligten Geschäftsgegner aus ${ }^{20}$ Die AGB des Authentisierungsnehmers können jedoch zur Ausformung des Rechtsscheintatbestandes dienen. Verbieten diese AGB die Weitergabe der Zugangsdaten und halten sich die meisten Nutzer daran, ist dies ein Indiz dafür, dass nach der Verkehrserwartung über den Account abgegebene Willenserklärungen dem Ersteller des Accounts zugerechnet werden.

299 Andere Stimmen kommen ohne ${ }^{21}$ oder durch eine abweichende dogmatische Begründung ebenfalls zur Haftung des Account-Inhabers bei Weitergabe der Zugangsdaten. Der Begründung, dass die Zugangsdaten als Legitimationsmerkmale ein dem mehrmaligen Auftreten unter fremdem Namen gleichwürdiges Vertrauen begründen 22 kann nicht zugestimmt werden. Der Erklärungsempfänger kann nicht erkennen, dass der Dritte handelt, sodass er kein schützenswertes Vertrauen in dessen Vertretungsmacht bilden kann. Die Anwendung der Anscheinsvollmach 23 erscheint fernliegend. Mangels Begründung kann jedoch nur allgemein auf die Ungeeignetheit der Anscheinsvollmacht eingegangen werden 24

19 LG Aachen, Urteil v. 15. 12.2006, 5 S 184/06 - NJW-RR 2007, 565; AG Saarbrücken, Urteil v. 15. 2.2008, 37 C 1251/06, Rn. 31.

20 Vgl. hierzu $B G H$, Urteil v. 7. 11.2001, VIII ZR 13/01 (ricardo.de) - BGHZ 149, 129, 135 f.: bei der Auslegung der Willenserklärung können die AGB des Plattformbetreibers subsidiär herangezogen werden.

21 OLG München, Urteil v. 5. 2. 2004, 19 U 5114/03 - NJW 2004, 1328, 1329.

22 So Hanau, Handeln unter fremder Nummer, S. 40.

23 Wie ohne weitere Begründung durch das OLG Schleswig, Beschluss v. 19. 7. 2010, 3 W 47/10 - CR 2011, 52.

24 Unten Rn. 378 


\section{Bildschirmtext (Btx)}

Dieser Lösungsweg über die Rechtsscheinvollmacht wurde bereits zum Bildschirmtext (Btx) ${ }^{25}$ vertreten ${ }^{26}$ Bei Anwendung dieser Ansicht haftet der Account-Inhaber bei der Weitergabe der Zugangsdaten dem Geschäftspartner auf das positive Interesse. Diese Ansicht begründen manche Gerichte mit der Anwendung der Anscheinsvollmacht 27 wobei die Argumente jedoch die Anwendung der allgemeinen Rechtsscheingrundsätze näher legen ${ }^{28}$ Stimmen der Literatur teilen die Meinung, dass der Anschlussinhaber nach den Grundsätzen der Anscheinsvollmacht hafte 29 Redeker vertritt die restriktive Auffassung, dass nur im kaufmännischen Verkehr für die Weitergabe der Zugangsdaten gehaftet werde ${ }^{30} \mathrm{Im}$ privaten Bereich überwiege der Schutz der Familie (Art. 6 Abs. 1 GG), sodass eine Haftung ausscheide ${ }^{31}$ Obwohl der Bildschirmtext-Anschluss auf eine Person registriert ist und zum Abschluss von Rechtsgeschäften dient, erfüllt er auch ein allgemeines Informationsbedürfnis, sodass bei privaten Haushalten wegen des Teilens des Anschlusses kein Rechtsscheintatbestand bezüglich des Handeln des Anschlussinhabers bestehe 32

Jedenfalls außerhalb des Btx kann diese Einschränkung nicht aufrecht erhalten werden 33 Passwortgeschützte Accounts mit Identifikationsfunktion, wie z.B. der eBay-Account ${ }^{34}$ können individuell einer Person zugeordnet werden. Für die Befriedigung des Informationsbedürfnis der Familienmitglieder ist der Internetzugang, nicht jedoch die Weitergabe der Zugangsda-

25 Zur technischen Funktionsweise von Btx unten Rn. 498

26 OLG Köln, Urteil v. 30. 4. 1993, 19 U 134/92 - CR 1993, 552; LG Koblenz, Urteil v. 17. 9. 1990, 3 S 78/90 - NJW 1991, 1360; LG Ravensburg, Urteil v. 13. 6. 1991, 2 S 6/91 - CR 1992, 472, 473; Lachmann, NJW 1984, 405, 408; Redeker, NJW 1984, 2390, 2394; a.A.: Borsum/Hoffmeister, NJW 1985, 1205, 1206 nur mittels eines sicheren Benutzeridentifikationssystems werde ein Rechtsschein hervorgerufen.

27 OLG Köln, Urteil v. 30.4. 1993, 19 U 134/92 - CR 1993, 552; LG Ravensburg, Urteil v. 13.6. 1991, 2 S 6/91 - CR 1992, 472, 473.

28 Unten Rn. 302

29 Lachmann, NJW 1984, 405, 408. Dogmatisch Begründung unklar bei Redeker, NJW 1984, 2390, 2393.

30 Ebd. 2394.

31 Ebd. 2394.

32 Ausführlich dazu unten Rn. 508

33 Rieder, S. 198. Gegen diese Ansicht im Anwendungsbereich des Btx: Paefgen, CR 1993, 559, 562; Kuhn, S. 221.

34 Dazu oben Rn. 64 
ten zu den Accounts erforderlich. Einschränkungen bei der Haftung für den Missbrauch von Zugangsdaten im familiären Bereich ergeben sich daher nicht aus Art. 6 Abs. 1 GG.

\section{Kritik}

302 Die Anwendung der Duldungsvollmacht stellt keine überzeugende dogmatische Herleitung der Haftung für den Missbrauch von Zugangsdaten nach deren Weitergabe dar. Der Geschäftsgegner kann nicht erkennen, dass ein Dritter gehandelt hat ${ }^{35}$ Er kann daher nicht darauf vertrauen, dass dieser für ihn nicht erkennbar handelnde Dritte Vertretungsmacht für den AccountInhaber hatte ${ }^{36}$ Für den Rechtsscheintatbestand ist der Schein einer Vertretungsmacht daher kein geeigneter Anknüpfungspunkt. Vielmehr muss sich der Rechtsscheintatbestand darauf beziehen, ob der Erklärungsempfänger schützenswert darauf vertrauen konnte, dass der Account-Inhaber gehandelt hat ${ }^{37}$ Bei der Duldungsvollmacht kann zwar im Gegensatz zur Anscheinsvollmach ${ }^{38}$ ein erstmaliges Auftreten die Haftung begründen ${ }^{39}$ dies kann jedoch mangels Erkennbarkeit für den Geschäftsgegner keine Haftung begründen ${ }^{40}$ Schon früh erkannte das $L G$ Ravensburg dies. Es bezeichnet die Haftung zwar als eine Haftung kraft Anscheinsvollmacht, es verwendet jedoch in der Begründung allgemeine Rechtsscheingrundsätze 41

\section{Lösung über die Übertragung des Rechtsgedankens des $\$ 172$ Abs. 1 $B G B$}

303 In Teilen der Literatur hat sich gegen die Anwendung der Duldungsvollmacht eine Ansicht gebildet, die das Ergebnis auf einer dogmatisch überzeugenderen Weise herzuleiten versucht. Die Rechtsscheinhaftung für den Missbrauch der Zugangsdaten bei deren Weitergabe lasse sich aus dem

35 Wiebe, Elektronische Willenserklärung, S. 426.

36 Dörner, AcP 202 (2002), 363, 389 f.

37 Unten Rn. 378

38 Oben Rn. 268

39 Oben Rn. $\overline{263}$

40 Herresthal, K\&R 2008, 705, 707; ders., in: Taeger/Wiebe, 21, 31.

41 LG Ravensburg, Urteil v. 13.6. 1991, 2 S 6/91 - CR 1992, 472, 473. 
Rechtsgedanken des $\S 172$ Abs. 1 BGB begründen 42 Zur Veranschaulichung der Meinung soll zunächst der ursprüngliche Anwendungsbereich des $§ 172$ Abs. 1 BGB sowie seine schrittweise Erweiterung auf die Blanketterklärungen dargestellt werden. Im Anschluss werden diese Grundsätze auf die Haftung für den Missbrauch von Zugangsdaten im Internet angewendet. Die Lösung über eine analoge Anwendung des § 172 Abs. 1 BGB kann sowohl in Zwei- als auch in Drei-Personen-Konstellationen angewendet werden.

\section{Ursprünglicher Anwendungsbereich des $§ 172$ Abs. 1 BGB}

Nach $\S 172$ Abs. 1 BGB ist derjenige, der einem Dritten eine Vollmachtsurkunde vorlegt, diesem gegenüber zur Vertretung des Ausstellers der Urkunde befugt. Es handelt sich dabei um eine gesetzlich kodifizierte Rechtsscheinhaftung 43

\section{a) Bedeutung des $\S 172$ Abs. 1 BGB}

Zweck des $\S 172$ Abs. 1 BGB ist es, zusammen mit $\S 171$ Abs. 1 BGB die nach außen getragene Innenvollmacht einer Außenvollmacht gleichzusetzen 44 Der zufällig gewählte Akt der Vollmachtskundgabe, ob sie gegenüber dem Vertreter oder dem Dritten erfolgt, soll keine Unterschiede in den Rechtsfolgen hervorrufen. Die Außenvollmacht bleibt bestehen, bis der Geschäftsherr sie gegenüber dem Dritten widerruft ( $\$ 170$ BGB). Die Rechtsscheintatbestände der $\S \S 171$ Abs. 1, 172 Abs. 1 BGB, die durch den actus contrarius zerstört werden können ( $\S 171$ Abs. 2, 172 Abs. 2 BGB) stellen diesen Gleichlauf sicher.

42 Insbesondere Oechsler, AcP 208 (2008), 565 sowie auch Faust, BGB AT ${ }^{3}, \S 26$ Rn. 41; Frensch, in: Prütting/Wegen/Weinreich ${ }^{8}$, § 172 BGB Rn. 8; J. Hoffmann, in: Leible/Sosnitza, Rn. 177; Kuhn, S. 217; Rieder, S. 158; Reese, S. 125; Schinkels, LMK 2011, 320461, 2 b bb; Schilken, in: Staudinger ${ }^{2009}$, § 172 BGB Rn. 8; Schramm, in: MüKo-BGB ${ }^{6}$, 172 Rn. 18; Spiegelhalder, S. 160; Sonnentag, WM 2012, 1614, 1617; Stöber, EWiR 2011, 521. Zur Botenkonstellation: Leible/Sosnitza, CR 2003, 344, 347.

43 Canaris, Vertrauenshaftung, S. 134.

44 Motive, S. 237; Mugdan, S. 484; Kindl, S. 12 f. 


\section{$\S 5$ Haftung bei Weitergabe}

306 Diese Gleichbehandlung von Außen- und Innenvollmacht überzeugt dort, wo frei zwischen den beiden Kundgabearten gewählt werden kann. Bei den ,typischen Innenvollmachten “45 in denen eine Erteilung als Außenvollmacht theoretisch oder praktisch nicht möglich ist, endet die systematische Parallele zur Außenvollmacht. Im Massenverkehr kann es daher zu unterschiedlichen Wertungen kommen ${ }^{46}$

307 Zwei Grundsätze lassen sich aus den Regelungen der §§ 170 ff. BGB herauskristallisieren. Zum einen haben schriftliche Willenserklärungen wie Urkunden keinen stärkeren Vertrauenstatbestand als mündliche Erklärungen ${ }^{47}$ Dies wird dadurch zum Ausdruck gebracht, dass das Vertrauen in eine Vollmachtsurkunde ( $\$ 172$ Abs. 1 BGB) genauso geschützt ist, wie die mündliche Erklärung über eine bestehende Vollmacht ( 171 Abs. 1 BGB). Zweitens handelt es sich bei den $\S \S 170$ ff. BGB um eine Haftung für die wissentliche Schaffung eines Rechtsscheintatbestandes 48

\section{b) Auslegung des $§ 172$ Abs. 1 BGB}

308 Als gesetzlich kodifizierte Rechtsscheinhaftung soll sich die Auslegung des $\S 172$ Abs. 1 BGB anhand der Voraussetzungen der allgemeinen Rechtsscheinhaftung 49 orientieren. Die allgemeinen Voraussetzungen sind der Rechtsscheintatbestand, dessen Zurechnung, die kausale Disposition des Vertrauenden sowie dessen Schutzwürdigkeit.

aa) Rechtsscheintatbestand

309 Die Vollmachtsurkunde muss zunächst die Schriftform des § 126 Abs. 1 BGB erfüllen ${ }^{50}$ Sie muss daher eigenhändig mit Namensunterschrift unter-

45 Dieser Begriff umfasst Vollmachten, die zur Vornahme von Rechtsgeschäften mit einem unbegrenzten Personenkreis bevollmächtigen, wie die Generalvollmacht und die Prokura. Der Begriff wurde geprägt durch Canaris, Vertrauenshaftung, S. 33.

46 Oechsler, AcP 208 (2008), 565, 575.

47 Canaris, JZ 1976, 132.

48 Rieder, S. 111.

49 Oben Rn. 226

50 Leptien, in: Soergel ${ }^{13}, \S 172$ BGB Rn. 2; Maier-Reimer, in: Erman ${ }^{13}, \S 172$ BGB Rn. 4; Schilken, in: Staudinger ${ }^{2009}$, § 172 BGB Rn. 1; Schramm, in: MüKo-BGB ${ }^{6}$ $\S 172$ Rn. 2. 
schrieben sein oder mittels notariell beglaubigten Handzeichens unterzeichnet werden. Diese Voraussetzung deutet der Wortlaut des $\S 172$ Abs. 1 BGB mit der Verwendung des Begriffes „Urkunde“ an. Systematisch ist daher die Gleichsetzung von Urkunde und der schriftlichen Form nach § 126 Abs. 1 BGB naheliegend. Teleologisch betrachtet stellt dies die Perpetuierung durch die Verkörperung des Willens des Geschäftsherren dar, auf das der Geschäftsgegner sein Vertrauen stützen kann 51 Die erforderliche Schriftform erfüllt zwei wichtige Komponenten bei der Etablierung des Rechtsscheintatbestandes. Zum einen erfüllt die Schriftform die Warnfunktion, dem Unterschreibenden die Ernsthaftigkeit der Unterschrift vor Augen zu führen 52 Der Vertrauende kann somit schützenswert von der Vollmachtsurkunde auf die Ernsthaftigkeit des Abschlusses von Rechtsgeschäften auf Seiten des Geschäftsherren vertrauen. Zum anderen hat die Schriftform darüber hinaus eine entscheidende Funktion bei der Überprüfung der Echtheit. Die Unterschrift als Teil der Handschrift einer Person ist ein für jede Person individuelles Sein-Merkmal ${ }^{53}$ Die Unterschrift kann zwar leicht nachgemacht werden. Fälschungen der Unterschrift können jedoch im Nachhinein aufgedeckt werden, sodass nur die Person selbst eine Vollmachtsurkunde mit der eigenhändigen Unterschrift herstellen kann. Das Schriftformerfordernis sorgt neben der Warnfunktion auch dafür, dass Vollmachtsurkunden nur schwer gefälscht werden können.

Ferner ist erforderlich, dass der Vertreter die Urschrift oder eine notarielle Ausfertigung ( $\$ 47$ BeurkG) der Vollmachtsurkunde vorlegt 54 Eine Fotokopie oder eine beglaubigte Abschrift reicht somit nach überwiegender Meinung nicht aus. Nach anderer Ansicht soll das Vertrauen in den mittelbaren Scheintatbestand der Kopie ausreichen, jedoch nur solange der Vertreter noch im Besitz des Originals ist 55 Die Voraussetzung, dass das Original oder eine notarielle Ausfertigung vorgelegt werden muss, entspricht schon dem natürlichen Verständnis der grammatikalischen Formulierung des $§ 172$ Abs. 1 BGB. Erforderlich ist demnach, dass ,eine Vollmachts-

\section{Schramm, in: MüKo-BGB 6 \$ 172 Rn. 2.}

52 Dazu etwa Faust, BGB AT ${ }^{3}, \S 8$ Rn. 1.

53 Oben Rn. 116

$54 R G$, Urteil v. 25.4. 1934, V 32/34 - JW 1934, 2394; BGH, Urteil v. 15. 10. 1987, III ZR 235/86 - BGHZ 102, 60, 63; Faust, BGB AT ${ }^{3}$, § 26 Rn. 28; H. Köhler, BGB $\mathrm{AT}^{37}, \S 11$ Rn. 40; Leptien, in: Soergel ${ }^{13}$, § 172 BGB Rn. 4; Maier-Reimer, in: $E_{r m a n}{ }^{13}, \S 172$ BGB Rn. 6; Oechsler, AcP 208 (2008), 565, 574; Schilken, in: Staudinger ${ }^{2009}, \S 172$ BGB Rn. 4; Schramm, in: MüKo-BGB 6 , § 172 Rn. 8.

Canaris, Vertrauenshaftung, S. 509. 
urkunde“ ausgehändigt wird und ,sie“ dem Dritten vorgelegt wird. Systematisch lässt sich diese Auslegung dadurch begründen, dass für den Vertretenen die Möglichkeit bestehen muss durch Rücknahme der Vollmachtsurkunde den Rechtsschein zu zerstören (vgl. § 172 Abs. 2 BGB). Würden Kopien oder beglaubigte Abschriften für den Rechtsschein genügen, könnte der Vertreter diese Möglichkeit der Zerstörung des Rechtsscheins durch unzählige Reproduktionen der Urschrift unterlaufen. Denn diese lassen keinen Rückschluss über den Verbleib des Originals sowie über den Fortbestand der Vollmacht zu $\sqrt[56]{6}$ Ferner stellt diese Voraussetzung sicher, dass der Vertretene die Vollmacht wirklich bereits erteilt hat und es ihm nicht kraft Zurückhaltens des Originals an einem Erklärungswillen fehlt ${ }^{57}$ Teleologisch betrachtet dient diese Voraussetzung der Etablierung eines starken Rechtsscheintatbestandes. Eine Urschrift oder eine notarielle Ausfertigung erwecken mehr Vertrauen als eine Kopie oder eine beglaubigte Abschrift, weil sie physisch einmalig sind. Der Besitz einer physisch einmaligen Sache ist ein geeigneter Rechtsscheinträger, wie systematisch $\S 1006$ Abs. 1 S. 1 BGB zeigt. Der Besitz der physisch einmaligen Vollmachtsurkunde ist ein zentraler Aspekt des Rechtsscheintatbestandes des $§ 172$ Abs. 1 BGB

311 Darüber hinaus muss der Vertreter sowie der Umfang der Vertretungsmacht auf der Urkunde benannt sein 58 Dieses Erfordernis wird behauptet, ohne es näher zu begründen. Aus dem Wortlaut des $§ 172$ Abs. 1 BGB ergibt sich diese Einschränkung nicht. Diese Voraussetzung folgt jedoch aus dem Sinn und Zweck des $\S 172$ Abs. 1 BGB. Als gesetzlich kodifizierter Rechtsscheintatbestand muss auch im Fall des $§ 172$ Abs. 1 BGB ein schützenswertes Vertrauen auf Seiten des Geschäftsgegner vorhanden sein. Dieses kann nur bestehen, wenn die Vollmacht nicht nur schriftlich verfasst, sondern auch durch Angabe des Umfangs hinreichend bestimmt ist. Diese Voraussetzungen schränken die vielfältigen Missbrauchsmöglichkeiten einer Vollmachtsurkunde ein. Die Vollmachtsurkunde kann der Vertreter beliebig oft wieder verwenden, um den Geschäftsherrn zu verpflichten. Er kann gegenüber einer Vielzahl von Personen zahlreiche Rechtsgeschäfte im Namen des Geschäftsherren abschließen. Durch das Erfordernis des Umfangs werden seine Möglichkeiten eingeschränkt. Erteilt der Geschäfts-

57 Oechsler, AcP 208 (2008), 565, 574.

$58 R G$, Urteil v. 14. 6. 1929, VII 561/28 - RGZ 124, 383, 363; Schilken, in: Staudinger $^{2009}$, § 172 BGB Rn. 1; Frensch, in: Prütting/Wegen/Weinreich ${ }^{8}, \S 172$ BGB Rn. 2; Valenthin, in: Bamberger/H. Roth ${ }^{3}$, § 172 BGB Rn. 4. 
herr eine sehr umfangreiche Vollmacht, erfüllt das Erfordernis, den Umfang zu bestimmen, wenigstens eine Warnfunktion. Dieses Erfordernis zeigt, dass für den Rechtsscheintatbestand eine Beschränkung der Missbrauchsmöglichkeit oder wenigstens eine Warnung vor möglichem, umfangreichem Missbrauch erforderlich ist.

Die Urkunde muss zudem echt sein 59 also vom erkennbaren Aussteller stammen. Diese Einschränkung ist im Wortlaut des § 172 Abs. 1 BGB angedeutet. Er fordert die Aushändigung der Vollmachtsurkunde durch den Vertretenen. Es sind zwar Fälle denkbar, in denen der Vollmachtgeber die Urkunde nicht selbst ausgestellt hat. Die Tatsache, dass sie von ihm stammen muss, soll jedoch die Echtheit der Urkunde sicherstellen. Dieses Erfordernis erfüllt die allgemeine Voraussetzung eines Rechtsscheintatbestandes, dass er sich auf das Verhalten des In-Haftung-Genommenen beziehen muss ${ }^{60}$ Fehlt es an einer Anknüpfung an das Verhalten des Geschäftsherren, kann seine Haftung nicht begründet werden.

Die Betrachtung der Ausformung des Rechtsscheintatbestandes des $§ 172$ Abs. 1 BGB zeigt, dass er aus mehreren Komponenten besteht. Die entscheidende Komponente ist, dass der Rechtsschein von dem Besitz der physisch einmaligen Vollmachtsurkunde als Rechtsscheinträger ausgeht. Die Vollmachtsurkunde sorgt durch das Schriftformerfordernis für eine Warnfunktion und eine hohe Fälschungssicherheit. Durch das Erfordernis, dass der Umfang benannt werden muss, werden Missbrauchsmöglichkeiten eingeschränkt oder wenigstens vor ihnen gewarnt.

\section{bb) Zurechenbarkeit}

Als Aushändigung wird nur die willentliche Übergabe zum Zwecke des Gebrauchmachens verstanden 61 Diese Auslegung ergibt sich unmittelbar aus dem natürlichen Wortsinn des „Aushändigens“, das die von einem Willensentschluss getragene Besitzübertragung beinhaltet. Dass die Überga-

$59 R G$, Urteil v. 23. 5. 1917, V 29/17 - RGZ 90, 273, 279; Schilken, in: Staudinger ${ }^{2009}$, $\S 172$ BGB Rn. 1; Schramm, in: MüKo-BGB 6 , § 172 Rn. 3.

60 Oben Rn. 230

$61 B G H$, Urteil v. 30. 5. 1975, V ZR 206/73 - BGHZ 65, 13; OLG Karlsruhe, Urteil v. 13.6.2006, 1 U 22/05 - ZIP 2005, 1633, 1634; Canaris, Vertrauenshaftung, S. 39; Faust, BGB AT ${ }^{3}, \S 26$ Rn. 30; Schilken, in: Staudinger ${ }^{2009}$, § 172 BGB Rn. 2; Schramm, in: MüKo-BGB6 ${ }^{6}$ \& 172 Rn. 4. 
be zum Zwecke des Gebrauchmachens erfolgen muss, zeigt systematisch die Voraussetzung, dass der Vertreter die Vollmachtsurkunde vorlegen muss ( 172 Abs. 1 a.E. BGB). Eine freiwillige Aushändigung ist systematisch betrachtet das Gegenteil einer abhandengekommenen Vollmachtsurkunde (vgl. § 935 Abs. 1 BGB), sodass eine gestohlene, verloren gegangene oder sonst abhandengekommene Vollmachtsurkunde dem Aussteller nicht zurechenbar ist 62

315 Das schuldhafte Ermöglichen des Abhandenkommens der Vollmachtsurkunde reicht somit nach $\S 172$ Abs. 1 BGB nicht aus ${ }^{63}$ Es ist jedoch zu erwägen, § 172 Abs. 1 BGB analog bei abhandengekommenen Vollmachtsurkunden anzuwenden ${ }^{64}$ Dazu müssten die Voraussetzungen einer Analogie vorliegen, nämlich eine planwidrige Regelungslücke und eine vergleichbare Interessenlage 65 Gegen das Vorliegen einer planwidrigen Regelungslücke spricht zunächst, dass in $\S 170$ ff. BGB nur Rechtsscheintatbestände, die eine willentliche Schaffung des Rechtsscheins verlangen 66 geregelt sind. Die Gesetzessystematik anderer Rechtsscheintatbestände zeigt ebenfalls, dass nur die willentliche Schaffung des Rechtsscheintatbestandes die Haftung begründet. Nach $\S 935$ Abs. 1 BGB ist ein gutgläubiger Erwerb nur möglich, wenn der Eigentümer die Sache willentlich aus der Hand gegeben hat. Kommt die Sache abhanden, selbst aufgrund leichter Fahrlässigkeit des Eigentümers, scheidet der gutgläubige Erwerb aus. Zwar lässt die Vollmachtsurkunde ihren Aussteller erkennen, sodass ihr eine Identifikationsfunktion zukommt ${ }^{67}$ Dies beschreibt jedoch nur eine sachlogische Notwendigkeit bei Stellvertretungskonstellationen und dementsprechende Aus-

62 Canaris, Vertrauenshaftung, S. 38 f.; ders., JZ 1976, 132, 133; Oechsler, AcP 208 (2008), 565, 577 sowie ohne Bezug zu § 935 Abs. 1 BGB BGH, Urteil v. 30. 5. 1975, V ZR 206/73 - BGHZ 65, 13; Frensch, in: Prütting/Wegen/Weinreich ${ }^{8}$, $\S 172$ BGB Rn. 3; Jauernig, in: Jauernig ${ }^{15}$, §§ 170-172 BGB Rn. 8; Schramm, in: MüKo-BGB $^{6}$ \& 172 Rn. 5.

63 BGH, Urteil v. 30.5. 1975, V ZR 206/73 - BGHZ 65, 13, 14 f.; Bous, RPfleger 2006, 357, 360; Canaris, Vertrauenshaftung, S. 38; ders., JZ 1976, 132; Kindl, S. 17; Schilken, in: Staudinger ${ }^{2009}, \S 172$ BGB Rn. 7; M. Wolf/Neuner ${ }^{10}$, 50 Rn. 78.

64 So im Ergebnis Coing, in: Staudinger ${ }^{11}$, §§ 171-172 BGB Rn. 6; Enneccerus/Nipperdey $^{15}$, § 188 I 1c; Gotthardt, S. 48; Hupka, S. 174; Isay, S. 239; Weinschenk, LZ 1931, 1310, 1311 sowie jüngst Spiegelhalder, S. 151; Stöber, JR 2012, 225, 227 f.

65 Unten Rn. 329 ff.

66 Canaris, Vertrauenshaftung, S. 32.

67 Stöber, JR 2012, 225, 228. 
formung des Rechtsscheintatbestandes. Eine erweiterte Zurechnung lässt sich damit nicht begründen.

Eine vergleichbare Interessenlage liegt ebenso nicht vor. $§ 172$ Abs. 1 BGB hat mehrere Betroffene. Der Geschäftsgegner hat ein Interesse daran, dass auch fahrlässig abhandengekommene Vollmachtsurkunden die Vollmacht des Vertreters begründen. Der Vertretene hingegen hat ein schützenswertes Interesse, dass die Rechtsscheinhaftung auf ein angemessenes Maß reduziert ist. Der von einer unterschriebenen Urkunde ausgehende Rechtsschein ist daher nicht grenzenlos. Sie muss unter anderem echt sein ${ }^{68}$ Dies kann der Geschäftsgegner genau so überprüfen wie das Abhandenkommen, nämlich durch Rückfrage beim Vertretenen. Bei einem fahrlässigen Abhandenkommen besteht daher nicht die gleiche Interessenlage wie bei einer willentlichen Übergabe.

Die Wertungen der wertpapierrechtlichen Vorschriften des § 935 Abs. 2 BGB lässt sich nicht auf $\S 172$ Abs. 1 BGB übertragen 69 Der Gesetzgeber wollte durch die $\S \S 170$ ff. BGB die Gleichstellung von Außen- und Innenvollmachten erreichen ${ }^{70}$ Im Massenverkehr bei ,typischen Innenvollmachten“ wie Generalvollmachten besteht dabei zwar ein erhöhtes Vertrauensbedürfnis ${ }^{71}$ Doch auch ,typische Innenvollmachten“, die für den Massenverkehr perpetuiert wurden, werden nicht wie Geld oder Inhaberpapiere gehandelt. Die dadurch erreichte Beschleunigung und Rechtssicherheit bedarf es bei Vertretergeschäften nicht. Die Wertung des § 935 Abs. 2 BGB kann nicht auf sie übertragen werden.

Eine allgemeine Risikoabgrenzung begründet die vergleichbare Interessenlage ebenfalls nicht. Der Geschäftsherr schaffe durch die Urkunde ein objektives Moment, das im Rechtsverkehr einen gewissen Schein hervorruft, sodass er dafür einzustehen habe ${ }^{72}$ Für diesen Rechtsschein habe der Aussteller einzustehen ${ }^{73}$ Diese allgemeinen Risikoabwägungen können jedoch dort nicht überzeugen, wo der Gesetzgeber - wie in § 172 Abs. 1 BGB - eine eindeutige Wertung über die Risikoverteilung getroffen hat. Diese gilt es zu respektieren.

68 Oben Rn. 312

69 A.A. Spiegelhalder, S. 151; Weinschenk, LZ 1931, 1310, 1311.

70 Oben Rn. 305

71 Canaris, Vertrauenshaftung, S. 33, 39; ders., JZ 1976, 132, 133 f.

72 Isay, S. 239.

73 Coing, in: Staudinger ${ }^{11}$, §§ 171-172 BGB Rn. 6. 
319 Die vergleichbare Interessenlage lässt sich auch nicht aus einer Übertragung der Wertung des $§ 370$ BGB schließen ${ }^{74}$ Selbst bei der Annahme, die Ermächtigung der Quittung gelte auch für abhandengekommene Quittungen, kann daraus kein systematischer Schluss für die Auslegung des § 172 Abs. 1 BGB gezogen werden. In dessen Wortlaut verhindert das Erfordernis des „Aushändigens“ diese Interpretation. Eine analoge Anwendung des $\S 172$ Abs. 1 BGB auf den Fall von abhandengekommenen Vollmachtsurkunden scheidet somit aus.

cc) Disposition im Vertrauen auf den Rechtsschein

320 Das Merkmal des $\S 172$ Abs. 1 BGB, dass die Vollmachtsurkunde dem Dritten vorgelegt wird, setzt zunächst voraus, dass dem Dritten die Urkunde zur sinnlichen Wahrnehmung unmittelbar zugänglich gemacht wird 75 Lesen muss der Dritte die Urkunde nicht 76 Systematisch lässt sich dieses Erfordernis mit der Gleichstellung zur Mitteilung einer Innenvollmacht an einen Dritten ( $\$ 171$ Abs. 1 BGB) begründen 77 Teleologisch betrachtet wird durch den Rechtsscheinträger der Urkunde sowie dem Vorlegen die Voraussetzungen des schützenswerten Vertrauens ausgeformt.

\section{dd) Gutgläubigkeit des Dritten}

321 Das Erfordernis der Gutgläubigkeit des Dritten ergibt sich aus dem systematischen Zusammenhang mit $§ 173$ BGB. Dem Geschäftsgegner schadet Kenntnis sowie fahrlässige Unkenntnis bezüglich des Erlöschen der Vollmacht 78 Dies ergibt sich aus dem Wortlaut des $§ 173$ BGB ,kennen müssen“, das, systematisch betrachtet, die fahrlässige Unkenntnis erfasst ( $\S 122$ Abs. 2, 276 Abs. 2 BGB). Zwar verweist $§ 173$ BGB nicht auf den

74 Canaris, JZ 1976, 132.

$75 R G$, Urteil v. 26. 11. 1903, VI 140/03 - RGZ 56, 63, 66; Urteil v. 10. 12. 1919, V 249/19 - RGZ 97, 273, 275; BGH, Urteil v. 15. 10. 1987, III ZR 235/86 - BGHZ 102, 60, 63; Schilken, in: Staudinger ${ }^{2009}$, 172 BGB Rn. 3; Leptien, in: Soergel ${ }^{13}$, $\S 172$ BGB Rn. 4.

76 A.A.: Frotz, S. 301; Kindl, S. 19.

77 Schilken, in: Staudinger ${ }^{2009}, \S 172$ BGB Rn. 3.

78 Schilken, in: Staudinger ${ }^{2009}$, § 173 BGB Rn. 2; Schramm, in: MüKo-BGB 6 , § 173 Rn. 3. 
Fall der ursprünglichen Unrichtigkeit der Vollmachtsurkunde. Diese Formulierung des Wortlauts ist als Redaktionsversehen anzusehen, sodass $\S 173$ BGB (analog) auf $\S 172$ Abs. 1 BGB angewendet werden kann 79

2. Anwendung des $\$ 172$ Abs. 1 BGB auf den Missbrauch von Zugangsdaten

Eine direkte oder entsprechende Anwendung des $\S 172$ Abs. 1 BGB auf

Fälle des Missbrauchs von Zugangsdaten im Internet ist in einigen Konstellationen möglich. Zunächst kann der Geschäftsherr dem Dritten anstatt eine Vollmachtsurkunde auszustellen, die Vollmacht in elektronischer Form erteilen. Die Voraussetzungen, dass die Vollmachtsurkunde der Schriftform nach $\S 126$ Abs. 1 BGB genügen muss, kann nur die qualifizierte elektronische Signatur erfüllen ( $\$ 126$ Abs. 3 BGB i.V.m. $\S 2$ Nr. 3 SigG) ${ }^{80}$ Diese Vollmacht in elektronischer Form kann auch dem Bevollmächtigten ausgehändigt werden. Zwar kann das Aushändigen nicht in der körperlichen Form des natürlichen Wortsinnes erfolgen. Gleichwohl kann ein Versenden per E-Mail als ebenbürtig eingestuft werden. Diese E-Mail kann der Bevollmächtigte auch der sinnlichen Wahrnehmung des Dritten unmittelbar zugänglich machen. Problematisch ist jedoch, dass eine elektronische Vollmacht nicht verkörperlicht ist. Die Perpetuierungsfunktion des Schriftformerfordernisses ist erfüllt, nicht jedoch die Anforderung der physischen Einmaligkeit. Dem Geschäftsherren fehlt die systematisch bedeutende Möglichkeit wieder in Besitz der Urkunde zu gelangen ( $\$ 172$ Abs. 2 BGB) und dadurch den Rechtsschein zu zerstören. Im Internet gibt es keinen Unterschied zwischen Original und Kopie 81 Dort sind vielmehr alle Wiedergaben einer Erklärung lediglich Kopien ${ }^{82}$ Ebenso wie bei $\S 172$ Abs. 1 BGB eine Kopie nicht ausreichend für die Rechtsscheinhaftung ist. ${ }^{83}$ reicht eine elektronische Vollmachtsurkunde nicht aus.

$79 R G$, Urteil v. 19.3. 1923, V 427/22 - RGZ 108, 125, 127; BGH, Urteil v. 8. 11. 1984, III ZR 132/83 - NJW 1984, 730; Enneccerus/Nipperdey ${ }^{15}$, § 188 I 2; Faust, BGB $\mathrm{AT}^{3}, \S 26$ Rn. 30, 27; Maier-Reimer, in: Erman ${ }^{13}$, § 173 BGB Rn. 2; Schilken, in: Staudinger ${ }^{2009}, \S 173$ BGB Rn. 7.

80 So auch Oechsler, AcP 208 (2008), 565, 570.

81 Roßnagel, MMR 2002, 67, 68; Spiegelhalder, S. 120.

82 Spiegelhalder, S. 120.

83 Ebd. S. 121 sowie oben Rn. 312 
323 Ein weiterer diskutierter, jedoch kaum vorkommender Fall ist, dass die Zugangsdaten auf einer schriftlichen Urkunde übergeben werden ${ }^{84}$ Diese Urkunde müsste dem Geschäftsgegner auch vorgelegt werden. Allein die Verwendung der Zugangsdaten im Authentisierungsvorgang reicht dafür nicht aus. Somit scheidet auch für den unwahrscheinlichen Fall, dass der Account-Inhaber dem Handelnden die Zugangsdaten auf einer schriftlichen Urkunde ausgehändigt hat, die Anwendung des § 172 Abs. 1 BGB aus. Lediglich Vollmachts-Attributzertifikate nach $\S 8$ Abs. 2 SigG haben eine vergleichbare Funktion wie Vollmachtsurkunden, sodass für diese $§ 172$ Abs. 1 BGB analog angewendet werden kann 85

3. Analoge Anwendung des $\$ 172$ Abs. 1 BGB auf verdeckte Blanketterklärungen

324 Die Auslegung des $\S 172$ Abs. 1 BGB hat dessen enge Voraussetzungen aufgezeigt. Angewendet auf das Internet können nur Vollmachts-Attributzertifikate diese strengen Anforderungen erfüllen. In der Rechtspraxis wurden die Voraussetzungen des $\S 172$ Abs. 1 BGB jedoch schrittweise durch die Anwendung auf offene und verdeckte Blankette aufgelockert.

Ein Blankett, auch Blanko genannt, ist eine schriftliche Erklärung, die zwar eine Unterschrift enthält, bei der jedoch bewusst mindestens ein inhaltlicher Punkt unvollständig ausgefüllt ist, damit ein Dritter die offene, auch als blank bezeichnete, Stelle ausfüllen kann. Ein Beispiel für ein Blankett ist ein vom Geschäftsherrn unterschriebener Kaufvertrag, bei dem der Vertreter nach Verhandlungen mit dem Käufer noch den ausgehandelten Kaufpreis einträgt, den der Geschäftsherr im Gegensatz zu allen anderen Teilen des Vertrags offen gelassen hat. Das Ausfüllen des Blanketts wird als „Perifizierung" bezeichnet ${ }^{86}$ Einhergehend mit dem Ausstellen des Blanketts ergeht regelmäßig die Erteilung einer „Ermächtigung“, das Blankett auszufüllen 87

326 Dogmatisch fällt die Einordnung des Blankettausfüllers schwer. Bei einer offenen Blanketterklärung erweckt der Besitz der Blankourkunde den Anschein, dass der Ausfüller eine der Vertretungsmacht ähnliche Ausfül-

84 Hanau, Handeln unter fremder Nummer, S. 34; ders., VersR 2005, 1215, 1218.

85 Rieder, S. $141 \mathrm{ff}$. ihm folgend Reese, S. 110.

86 Begriff geprägt durch Canaris, Vertrauenshaftung, S. 55.

87 Schramm, in: MüKo-BGB 6 § 172 Rn. 14. 
lungsermächtigung hat. Bei der verdeckten Blanketterklärung hingegen erscheint er dem Erklärungsempfänger als Bote. Im Ergebnis stellt sich die Blanketterklärung als arbeitsteilig erstellte Willenserklärung dar 88

Bei der abredewidrigen Ausfüllung ergibt sich das gleiche Problem, wie beim Missbrauch von Zugangsdaten im Internet, nämlich ob der Geschäftsherr durch das Handeln des Dritten rechtsgeschäftlich verpflichtet wird. Gesetzlich ist die Frage des abredewidrig ausgefüllten Blankos für Wechsel in Art. 10 WechselG und für Schecks Art. 13 ScheckG geklärt ${ }^{89}$ In älterer Literatur wird deren analoge Anwendung diskutiert ${ }^{90}$ Schecks und Wechsel bedürfen jedoch wie alle Wertpapiere einer erhöhten Umlauffähigkeit 91 Wertpapierrechtliche Vorschriften bieten wegen dieser fundamental unterschiedlichen Interessenlage keine Grundlage für eine Analogie zu Blanketterklärungen im Bürgerlichen Recht 92

$\mathrm{Zu}$ erwägen ist daher, ob die abredewidrige Blankettvervollständigung über eine analoge Anwendung des $\S 172$ Abs. 1 BGB erfolgen kann. Dies wird häufig über zwei Schritte gelöst. Dabei wird nicht nur die Analogie in mehreren Schritten begründet, sondern die tatbestandlichen Voraussetzungen werden auch schrittweise aufgeweicht.

\section{a) Exkurs: Voraussetzungen einer analogen Anwendung}

Bei der analogen Anwendung einer Norm auf einen anderen Fall im Rahmen der Rechtsfortbildung stellt sich die Frage, unter welchen Voraussetzungen dies möglich ist. Dazu muss zunächst eine Unvollständigkeit des Gesetzes oder der Rechtsordnung vorliegen 93 Ob eine Unvollständigkeit im Gesetz eine Lücke darstellt, ist bei Gesetzen am Plan des historischen Gesetzgebers zu beurteilen ${ }^{94}$ Stellt sich heraus, dass diese Unvollständig-

88 Zum Streit zwischen Boten-, Ermächtigungs- und Vertretertheorie vgl. Binder, AcP 207 (2007), 155, 160 ff.; Kindl, S. 124 ff.; Gerd Müller, AcP 181 (1981), 515, $518 \mathrm{ff}$.

89 Bork $^{3}$, Rn. 1650.

90 K. Feldmann, S. 45 ff.; P. Fischer, S. 207 ff.

91 Dieses Bedürfnis kommt auch in $\S 935$ Abs. 2 BGB zum Ausdruck.

92 Canaris, Vertrauenshaftung, S. 62; G. Fischer, S. 64 f.; Kindl, S. 129 Fn. 45; Gerd Müller, AcP 181 (1981), 515, 145 ff.; Wurm, JA 1986, 577, 581.

93 Canaris, Lücken im Gesetz ${ }^{2}$, S. 25.

94 Pawlowski, Methodenlehre ${ }^{3}$, Rn. 463. 
keit mit dem Plan des Gesetzgebers nicht vereinbar ist, handelt es sich um eine Lücke95

330 Es sind verschiedene Arten von Lücken zu unterscheiden. Bei der Gesetzeslücke ${ }^{96}$ auch Normlücke $9^{97}$ oder Formulierungslücke $e^{98}$ genannt, ist die Regelung einer Norm in sich oder im Zusammenhang mit den Gesetz, in dem sie geregelt ist, lückenhaft. Eine Gesetzeslücke liegt nur vor, wenn nach dem historischen Konzept des Gesetzgebers eine Regelung des Falls den anderen geregelten Fällen entspricht, aber nicht vorgesehen wurde 99

331 Die zweite Form der Lücke ist die Regelungslücke ${ }^{100}$ auch Wertungsmangel genannt ${ }^{101}$ Regelungslücken liegen vor, wenn ein Gesetz an sich abschließend ist, ein anderer Teil der Rechtsordnung dadurch jedoch eine Unvollständigkeit aufweist ${ }^{102}$ Dabei ist zu unterscheiden zwischen anfänglichen, bei denen der Gesetzgeber schon bei Erlass von einer Lücke ausgeht, und nachträglichen Regelungslücken, die durch Änderungen anderer Gesetze oder technologischen Fortschritt entstehen ${ }^{103}$ Die erste Voraussetzung ist somit eine planwidrige Regelungslücke.

332 Entscheidende Voraussetzung für die Anerkennung einer Analogie nach der Interessenjurisprudenz ist, dass das Recht ansonsten widersprüchlich wäre und dem Grundsatz der Gleichbehandlung nicht gerecht würde ${ }^{104}$ Der Gleichbehandlungsgrundsatz ist dabei nicht nur eine Voraussetzung für die Analogiebildung sondern auch ein Instrument zur Lückenfindung ${ }^{105}$ Denn häufig steht am Anfang der Überlegung eines Analogieschlusses, dass eine Regelung für einen ähnlich gelagerten Fall vorhanden ist, dessen Wertung übertragen werden soll. Entscheidend für die Gleichheit ist die Überein-

95 Larenz/Canaris ${ }^{3}$, S. 192.

96 Ebd. S. 193.

97 Canaris, Lücken im Gesetz ${ }^{2}$, S. 59; Pawlowski, Methodenlehre ${ }^{3}$, Rn. 464.

98 Zippelius $^{11}$, S. 52.

99 Larenz/Canaris ${ }^{3}$, S. 196.

100 Canaris, Vertrauenshaftung, S. 60; Larenz/Canaris $^{3}$, S. 193; Pawlowski, Methodenlehre $^{3}$, Rn. 467.

101 Zippelius $^{11}$, S. 52.

102 Larenz/Canaris $^{3}$, S. 196.

103 Canaris, Lücken im Gesetz ${ }^{2}$, S. 135 f.; Larenz/Canaris $^{3}$, S. 200; Pawlowski, Methodenlehre $^{3}$, Rn. 470.

104 Larenz/Canaris $^{3}$, S. 195 f.; Pawlowski, Methodenlehre ${ }^{3}$, Rn. 475.

105 Canaris, Lücken im Gesetz ${ }^{2}$, S. 57; Zippelius ${ }^{11}$, S. 53. 
stimmung der Interessen bezüglich des Telos der Regelung ${ }^{106}$ Die zweite Voraussetzungen einer Analogie ist somit die vergleichbare Interessenlage.

Der Analogie verwandt ist das argumentum a maiore ad minus, der ErstRecht-Schluss ${ }^{107}$ Bei der Begründung einer Analogie ist stets auch die Möglichkeit eines argumentum e contrario, ein Umkehrschluss, in Betracht zu ziehen ${ }^{108}$ Aus der Tatsache, dass der Gesetzgeber eine Sache geregelt hat, kann bei entsprechendem gesetzgeberischen Willen geschlossen werden, dass er den nicht geregelten Fall nicht unter die Regelung fassen wollte.

b) Erster Schritt: offene Blanketterklärungen

Der erste Schritt ist die analoge Anwendung des § 172 Abs. 1 BGB auf 334 offene Blanketterklärungen. Bei einem offenen Blankett füllt der Ausfüllende dieses im Beisein des Dritten aus. Für die Analogie bedarf es einer planwidrigen Regelungslücke sowie einer vergleichbaren Interessenlage 109 Der Fall der Blanketterklärung sowie deren abredewidrige Ausfüllung ist gesetzlich nicht geregelt. Somit liegt eine planwidrige Lücke in Form einer nachträglichen Regelungslücke ${ }^{110}$ mangels gesetzgeberischer Regelung vor.

Ferner muss die Interessenlage vergleichbar sein. Die zentralen Merkmale des Rechtsscheintatbestandes des $§ 172$ Abs. 1 BGB erfüllt das offene Blankett. Das Blankett ist wie die Vollmachtsurkunde ein physisch einmaliges Objekt, dessen Besitz ein starker Rechtsscheinträger ist ${ }^{111}$ Ferner ist durch das zu übertragende Schriftformerfordernis nicht nur die Warnfunktion, sondern durch die Unterschrift als Sein-Merkmal auch eine gewisse Fälschungssicherheif ${ }^{112}$ erreicht.

Das offene Blankett unterscheidet sich jedoch von der Vollmachtsurkun336 de dadurch, dass der Dritte nicht als Vertreter genannt wird und somit der Umfang seiner Vollmacht nicht benannt ist 113 Aus Sicht des Dritten ist

106 Larenz/Canaris $^{3}$, S. 202 f.

107 Ebd. S. 208.

108 Canaris, Lücken im Gesetz ${ }^{2}$, S. 44; Larenz/Canaris $^{3}$, S. 209.

109 Oben Rn. 329 ff.

110 Oben Rn. 331

111 Vgl. Kindl, S. 129; Gerd Müller, AcP 181 (1981), 515, 524.

112 Oben Rn. 309

113 Dazu oben Rn. 311 
die offene Stelle im Blankett jedoch ein Indiz dafür, dass der Ausfüller eine gewisse Ausfüllungsermächtigung von dem Urkundenaussteller erhalten hat ${ }^{114}$ Die Anforderungen an den Rechtsscheintatbestand werden dadurch leicht abgeschwächt ${ }^{115}$ Eine Rechtsscheinhaftung trete beim offenen Blankett nur ein, wenn das Ausfüllen im Rahmen des Üblichen erfolgt 116 Demgegenüber sind jedoch die Missbrauchsmöglichkeiten erheblich eingeschränkt. Da es sich beim Blankett um ein physisch einmaliges Objekt handelt, kann es nur einmal ausgefüllt werden. Im Gegensatz zur Vollmachtsurkunde kann der Dritte das offene Blankett nur für ein Rechtsgeschäft verwenden. Eine Benennung des Umfangs der Ausfüllungsermächtigung ist somit weniger entscheidend, weil der Geschäftsherr durch das Blankett bereits einen Rahmen vorgegeben hat. Diese Interessenlage ist zwar nicht identisch, die Gemeinsamkeiten sind jedoch so groß, dass sie vergleichbar sind ${ }^{117} \S 172$ Abs. 1 BGB kann daher analog auf offene Blanketterklärungen angewendet werden.

\section{c) Zweiter Schritt: verdeckte Blanketterklärungen}

337 Bei einer verdeckten Blanketterklärung füllt der Ausfüllende das Blankett aus, bevor er es dem Dritten übergibt. Aus dessen Sicht liegt daher eine Erklärung des Unterschreibenden vor, die der scheinbare Bote - der eigentlich Ausfüllender ist - übergibt. Die analoge Anwendung des § 172 Abs. 1 BGB wird unterschiedlich begründet ${ }^{118}$ Zum einen wird die analoge Anwendung des $\S 172$ Abs. 1 BGB auf offene Blanketterklärungen analog auf die verdeckte Blanketterklärung angewandt. Bei dem verdeckten Blankett dürfe der Dritte nicht schlechter stehen als beim offenen 119 Es soll nicht vom Zufall abhängen, ob das Blankett offen oder verdeckt ausgefüllt wor-

114 Oechsler, AcP 208 (2008), 565, 569; Canaris, Vertrauenshaftung, S. 59.

115 Gerd Müller, AcP 181 (1981), 515, 524.

116 Canaris, Vertrauenshaftung, S. 59.

117 Oechsler, AcP 208 (2008), 565, 569; Wurm, JA 1986, 577, 578.

118 Hanau, Handeln unter fremder Nummer, S. 34 wendet $§ 172$ Abs. 1 BGB direkt an, geht dabei jedoch fälschlicherweise davon aus, dass „§ 172 BGB von einer Blanketturkunde [spricht]“.

119 Canaris, Vertrauenshaftung, S. 65; Kindl, S. 132; Oechsler, AcP 208 (2008), 565, 569; Wurm, JA 1986, 577, 579. Einschränkend Gerd Müller, AcP 181 (1981), 515, 526: der Geschäftspartner dürfe jedoch nicht besser dastehen als beim offenen Blankett. 
den ist. Dagegen spreche, dass die offene Blanketterklärung ein fiktiver Fall sei, der keine Rückschlüsse für den praxisrelevanten Fall der verdeckten Blanketterklärung zulasse ${ }^{120}$ Methodisch lässt sich jedoch auch der ErstRecht-Schluss zu einem fiktiven Fall begründen ${ }^{121}$ Ferner kommen Fälle offener Blanketterklärungen in der Praxis durchaus vor ${ }^{122}$

Zum anderen wird die Erfassung der verdeckten Blanketterklärungen mit einer analogen Anwendung des $\S 172$ Abs. 2 BGB begründet, ohne auf die offenen Blankette einzugehen ${ }^{123}$ Die Wahl des zweiten Absatzes von $\S 172$ BGB scheint jedoch nicht mit einer abweichenden Wertung verbunden zu sein. Bei beiden Absätzen des § 172 BGB handelt es sich um zwei Seiten einer Medaille. Nach $\S 172$ Abs. 1 BGB haftet der Aussteller der Urkunde für das Ausstellen und Aushändigen. Nach $\S 172$ Abs. 2 BGB haftet er jedoch nur so lange, wie die Urkunde im Umlauf ist.

Vereinzelt wird die Haftung für das abredewidrig ausgefüllte Blankett aus einer analogen Anwendung des $\S 172$ Abs. 1 BGB abgelehnt ${ }^{124}$ Der Geschäftsherr erkenne nicht, dass es sich um ein Blankett handele, sodass er kein Vertrauen in eine bestehende Vertretungsmacht wie bei der Vollmachtsurkunde entwickeln könne 125

Unabhängig von dem gewählten dogmatischen Weg soll nachfolgend untersucht werden, ob ein verdecktes Blankett entsprechend § 172 Abs. 1 BGB zu behandeln ist. Eine planwidrige Regelungslücke liegt ebenso wie beim offenen Blanket ${ }^{126}$ in Form einer nachträglichen Regelungslücke ${ }^{127}$ vor. Es stellt sich daher die Frage, ob die Interessenlage vergleichbar ist. Gemeinsam mit der Vollmachtsurkunde hat das verdeckte Blankett, dass von dem Besitz des Blanketts als physisch einmaliges Objekt ein starker Rechtsschein ausgeht. Ferner ist ebenso wie bei der Vollmachtsurkunde durch das zu übertragende Schriftformerfordernis nicht nur eine Warnfunktion erfüllt, sondern die Fälschung von Blanketts wird erheblich erschwert ${ }^{128} \mathrm{Im}$

120 Reinicke/Tiedtke, JZ 1984, 550, 552.

121 Kindl, S. 132.

122 Vgl. dazu die Sachverhalte von BGH, Urteil v. 12. 1. 1984, IX ZR 83/82 - NJW 1984, 798; Urteil v. 29. 2. 1996, IX ZR 153/95 - BGHZ 132, 119.

$123 B G H$, Urteil v. 11. 7. 1963, VII ZR 120/62 - BGHZ 40, 65, 68; Urteil v. 25. 11. 1963, II ZR 54/61 - BGHZ 40, 297, 304 f., zustimmend Flume ${ }^{4}$, § 232 c).

124 G. Fischer, S. 70; Reinicke/Tiedtke, JZ 1984, 550, 552.

125 Reinicke/Tiedtke, JZ 1984, 550, 552.

126 Oben Rn. 334

127 Oben Rn. 331

128 Vgl. oben Rn. 309 
Gegensatz zur Vollmachtsurkunde offenbart das verdeckte Blankett weder die Vertretungssituation noch den Umfang einer Ausfüllungsermächtigung. Daher muss das verdeckte Blankett als Urkunde bereits im Kern vor der Aushändigung geschaffen sein $\sqrt{129}$ Ein Vertragsformular ${ }^{130}$ oder eine „Oberschrift ${ }^{131}$ reicht dafür nicht aus. Nur eine unterschriebene, teilweise inhaltlich offen gelassene Willenserklärung begründet den Rechtsschein. Somit ist zwar der Umfang der Ausfüllungsermächtigung für den Empfänger nicht erkennbar, das hinter dem Erfordernis stehende Prinzip ist jedoch erfüllt. Die Missbrauchsmöglichkeiten sind beim verdeckten Blankett jedoch durch zwei Aspekte beschränkt. Da das Blankett bereits im Kern geschaffen sein muss, ist der Umfang, in dem der Missbrauch erfolgen kann, nicht genau so präzise aber ähnlich wirksam beschränkt, wie bei einer Vollmachtsurkunde. Hinzu kommt, dass das physisch einmalige Blankett im Original nur einmal ausgefüllt werden kann. Ein vielfacher Missbrauch wie bei der Vollmachtsurkunde ist somit nicht möglich. § 172 Abs. 1 BGB kann somit analog auf verdecke Blanketturkunden angewendet werden.

341 Bei der Schutzwürdigkeit des Vertrauenden ist es erforderlich, dass der Geschäftsgegner nicht erkennt, dass es sich um eine Blanketturkunde handelt. Füllt er die Urkunde selbst aus, tritt wegen seiner Bösgläubigkeit keine Rechtsscheinhaftung ein ${ }^{132}$ Entgegen der vom $R Q^{133}$ sowie Teilen der Literatur ${ }^{134}$ vertretenen Meinung kommt eine Anfechtung der ausgefüllten Blanketterklärung wegen des abredewidrigen Ausfüllens nicht in Betracht ${ }^{135}$ Das Aushändigen des Blanketts an den Ausfüller erfolgt lediglich

129 Gerd Müller, AcP 181 (1981), 515, 528.

$130 B G H$, Urteil v. 10.3. 1976, VIII ZR 210/74 - WM 1976, 507, 508.

$131 B G H$, Urteil v. 20.11.1990, XI ZR 107/89 - BGHZ 113, 48, 53 f. Bei einer „Oberschrift“ ist im Gegensatz zur Unterschrift das Dokument oben signiert. Die „Oberschrift“ kann nicht wie eine untenstehende Signatur die Abschlussfunktion erfüllen.

$132 B G H$, Urteil v. 12. 1. 1984, IX ZR 83/82 - NJW 1984, 798, 799; Urteil v. 29. 2. 1996, IX ZR 153/95 - BGHZ 132, 119, 128; a.A. Reinicke/Tiedtke, JZ 1984, 550, 552 : Rechtsscheinhaftung höhenmäßig begrenzt auf die abredegemäße Summe.

$133 R G$, Urteil v. 25. 9. 1922, VI 78/22 - RGZ 105, 183, 185.

134 Enneccerus/Nipperdey ${ }^{15}$, § 167 II 1; Enneccerus/H.Lehmann ${ }^{15}$, § 191 II 3; Gerd Müller, AcP 181 (1981), 515, 541; Pawlowski, JZ 1997, 309, 312; Reinicke/Tiedtke, JZ 1984, 550, 552; Siegel, Blanketterklärung, S. 45 ff.; ders., AcP 111 (1914), 1, 95; v. Tuhr, S. 415, $571 \mathrm{f}$.

135 Armbrüster, in: MüKo-BGB ${ }^{6}$ § 119 Rn. 55; Canaris, Vertrauenshaftung, S. 60; Leipold, BGB I: Einführung und Allgemeiner Teil ${ }^{7}, \S 23$ Rn. 30; Medicus $^{10}$, Rn. 913; Schilken, in: Staudinger ${ }^{2009}$, § 172 BGB Rn. 8; Schramm, in:MüKo-BGB ${ }^{6}$. 172 
mit dem Motiv, dass dieses abredegemäß ausgefüllt wird ${ }^{136}$ Motivirrtümer stellen jedoch keinen Anfechtungsgrund dar ${ }^{137}$ Ferner sprechen ein systematischer Gegenschluss zu $§ 172$ Abs. 2 BGB (analog) sowie der Zweck des Verkehrsschutzes durch Rechtsscheinhaftung gegen die Möglichkeit zur Anfechtung.

\section{d) Kein dritter Schritt: Der Kreditkartenmissbrauch}

Oechsler behauptet, dass beim Kreditkartenmissbrauch die Voraussetzungen des $\S 172$ Abs. 1 BGB noch weiter aufgelockert werden: Bereits der Besitz der Kreditkarte oder sogar nur die darauf enthaltenen Informationen würden zur Rechtsscheinhaftung ausreichen ${ }^{138}$ Zwar ist im Telefonoder Mail-Order-Verfahren noch nicht einmal der Besitz der Kreditkarte erforderlich, sondern lediglich das Wissen der Informationen auf der Karte reicht dabei zur Legitimation aus ${ }^{139}$ Dies betrifft jedoch eine vom Missbrauch von Zugangsdaten im Internet grundsätzlich verschiedene Konstellation. Beim Mail-Order-Verfahren benutzt der Dritte die Informationen auf der Kreditkarte des Inhabers, um bei einem Vertragsunternehmer eine Zahlung, die das Acquiring-Unternehmen ${ }^{140}$ garantiert, auszulösen. Vergleichbar mit der Frage der Haftung des Missbrauchs von Zugangsdaten im Internet, wäre die Frage, ob der Karteninhaber dem Acquiring-Unternehmen für den Missbrauch nach Rechtsscheingrundsätzen haftet. Der Karteninhaber haftet jedoch nicht ${ }^{141}$ Es fehlt an einem mit der Vollmachtsurkunde in sei-

Rn. 17. Implizit auch: BGH, Urteil v. 11. 7. 1963, VII ZR 120/62 - BGHZ 40, 65, 68; Urteil v. 25. 11. 1963, II ZR 54/61 - BGHZ 40, 297, 304.

136 So auch Canaris, Vertrauenshaftung, S. 60, 66.

137 Bork $^{3}$, Rn. 830 m.w.N.

138 Oechsler, AcP 208 (2008), 565, 570.

$139 B G H$, Urteil v. 16.4.2002, XI ZR 375/00 - BGHZ 150, 286, 297 ff.; Urteil v. 13. 1. 2004, XI ZR 479/02 - BGHZ 157, 256, 263. Kritisch dazu Meder, WM 2002, 1993, $1995 \mathrm{f}$.

140 Bei Kreditkarten gibt es regelmäßig vier Beteiligte: den Kreditkarteninhaber, den Emittenten der Kreditkarte, das Acquiring-Unternehmen sowie die Vertragshändler. Früher waren Emittent und Acquiring-Unternehmen häufig eine Person, die als Kreditkartenunternehmen bezeichnet wurde. Dazu Jungmann, in: Langenbucher/ Bliesener/Spindler, Kap. 6 Rn. 2 ff.; Martinek, in: Schimansky/BuntelLwowski ${ }^{4}$, $\S 67$ Rn. 2.

141 Langenbucher, S. 261; zustimmend BGH, Urteil v. 16. 4. 2002, XI ZR 375/00 BGHZ 150, 286, 292. 
ner physischen Einmaligkeit vergleichbaren Rechtsscheinträger, der einen Rechtsscheintatbestand begründen kann ${ }^{142}$

343 Beim Mail-Order-Verfahren besteht eine Haftung zwischen anderen Beteiligten. Das Acquiring-Unternehmen haftet dem Vertragspartner unter den in den AGB vereinbarten Voraussetzungen. Durch die vertraglichen Vereinbarungen zwischen dem Acquiring-Unternehmen und dem Vertragspartner entsteht ein abstraktes Schuldversprechen ( $\$ 780$ BGB) in den festgelegten Fällen ${ }^{143} \mathrm{Ob}$ sich die Haftung aus allgemeinen Risikoerwägungen ${ }^{144}$ oder aus einer analogen Anwendung des $§ 172$ Abs. 1 BGB ${ }^{145}$ ergibt, kann hier dahinstehen. Die für den Missbrauch der Zugangsdaten entscheidende Perspektive ist die des Kreditkarteninhabers. Er haftet dem Acquiring-Unternehmen bei missbräuchlicher Verwendung im Telefon- und Mail-OrderVerfahren nicht nach Rechtsscheingrundsätzen. Die Haftung des AcquiringUnternehmens beim Missbrauch der Kreditkarte stellt somit keinen dritten Schritt der Aufweichung der Voraussetzungen des $§ 172$ Abs. 1 BGB dar, die zur Begründung der Haftung des Account-Inhabers beim Missbrauch der Zugangsdaten relevant wäre.

e) Analoge Anwendung des $\S 172$ Abs. 1 BGB auf den Missbrauch von Zugangsdaten im Internet

344 Es stellt sich somit die Frage, ob der Missbrauch von Zugangsdaten im Internet über eine analoge Anwendung des $\S 172$ Abs. 1 BGB gelöst werden kann. Da die hier untersuchte Lösung nur den Fall der rein wissensbasierten Authentisierung zu lösen versucht, beschränken sich die nachfolgenden Ausführungen auf diese Authentisierungsmethode. Dabei wird nachfolgend untersucht, ob die Voraussetzungen für eine analoge Anwendung des $§ 172$ Abs. 1 BGB auf den Fall des Missbrauchs von Zugangsdaten im Internet erfüllt sind. Von einem dogmatischen Weg über die analoge Anwendung der analogen Anwendung des $\S 172$ Abs. 1 BGB auf die verdeckten Blanketterklärung ${ }^{146}$ ist Abstand zu nehmen. Weil bei der analogen Anwendung der verdeckten Blanketterklärung auf die Haftung für den Missbrauch von

142 In diese Richtung auch Langenbucher, S. 261.

$143 B G H$, Urteil v. 16. 4. 2002, XI ZR 375/00 - BGHZ 150, 286, 294.

144 Ebd. 297.

145 Martinek, in: Schimansky/Bunte/Lwowski ${ }^{4}$, § 67 Rn. 40.

146 Wie ihn Oechsler, AcP 208 (2008), 565, 578 ff. beschreitet. 
Zugangsdaten im Internet die Gefahr besteht, dass der Anwendungsfall sich $\mathrm{zu}$ weit von der ursprünglichen Norm entfernt, werden die Voraussetzungen der analogen Anwendung von $\S 172$ Abs. 1 BGB, nämlich die planwidrige Regelungslücke und die vergleichbare Interessenlage ${ }^{147}$ geprüft. Dabei werden jedoch die Wertungen der analogen Übertragung auf das offene und verdeckte Blankett, welche Tatbestandsmerkmale verzichtbar sind, berücksichtigt. Eine planwidrige Unvollständigkeit des Gesetzes liegt in Form einer nachträglichen Regelungslücke $e^{148}$ vor. Ob eine vergleichbare Interessenlage vorhanden ist, soll für den Rechtsscheintatbestand sowie für die Zurechnung untersucht werden.

\section{aa) Rechtsscheintatbestand}

Neben der planwidrigen Regelungslücke müsste eine vergleichbare Interessenlage vorliegen. Häufig wird pauschal behauptet, mit den Zugangsdaten gebe der Account-Inhaber ein Legitimationsmittel aus der Hand, das im Rechtsschein der Vollmachtsurkunde vergleichbar sei ${ }^{149}$ Bei dieser Behauptung trifft zwar zu, dass die Zugangsdaten den Handelnden gegenüber dem Authentisierungsnehmer legitimieren, ähnlich wie eine Vollmachtsurkunde den Vertreter legitimiert. Diese Legitimationsfunktion sagt jedoch nichts über die Stärke des Rechtsscheins aus.

Zunächst ist für eine vergleichbare Interessenlage entscheidend, ob von beiden Konstellationen ein ähnlich starker Rechtsschein ausgeht. Bei der Vollmachtsurkunde geht dieser Schein von dem Besitz eines physisch einmaligen Objekts aus ${ }^{150}$ Bei einer rein wissensbasierten Authentisierung kommt jedoch eine Besitz-Komponente nicht zum Einsatz. Dabei wird nur das Wissen um ein Geheimnis abgefragt, das unendlich teilbar ist und somit die gegenteilige Eigenschaft zur physischen Einmaligkeit besitzt. Selbst eine potentiell zu erwartende Geheimhaltung des Geheimnisses verstärkt den vom Wissen um das Geheimnis ausgehenden Rechtsschein nicht in einem vergleichbaren Maße. Wegen der unendlichen Teilbarkeit des Wissens und der fehlenden Möglichkeit des Account-Inhabers die Teilung des Wissens sowie das erstmalige In-Erfahrung-Bringen durch einen Dritten zu bemer-

147 Oben Rn. 329

148 Dazu oben Rn. 331

149 J. Hoffmann, in: Leible/Sosnitza, Rn. 177; Sonnentag, WM 2012, 1614, 1617.

150 Oben Rn. 310 
ken, kann er das Wissen im Gegensatz zu dem Besitz an einer physisch einmaligen Sache schlechter bis gar nicht kontrollieren. Der Rechtsscheinträger des Besitzes, der entscheidender Bestandteil des Rechtsscheintatbestandes des $\S 172$ Abs. 1 BGB ist, liegt somit beim Missbrauch von $\mathrm{Zu}$ gangsdaten im Internet nicht vor.

347 Eine zweite entscheidende Komponente des Rechtsscheintatbestandes des $\S 172$ Abs. 1 BGB liegt ebenfalls nicht vor: das Schriftformerfordernis 151 Beide Funktionen, die die Schriftform bei der Vollmachtsurkunde erfüllen soll, sind beim Missbrauch von Zugangsdaten im Internet nicht gegeben. Zum einen ist eine Warnfunktion bei Accounts im Internet nicht gegeben. Sie zu erstellen, eröffnet zunächst nur die Möglichkeit zur Kontaktaufnahme 152 Die Weitergabe der Zugangsdaten hat ebenfalls nicht eine mit einer Vollmachtsurkunde vergleichbare Warnfunktion. Denn die Unterschrift unter ein konkretes, den Unterzeichner verpflichtendes Dokument warnt ihn deutlicher vor den Konsequenzen als das einfache Mitteilen von Wissen über die Zugangsdaten. Ferner muss bei der Weitergabe kein Bezug zu einem Rechtsgeschäft bestehen. Der Account-Inhaber kann den Dritten bitten den Account auf neue Nachrichten zu überprüfen oder ihm die Zugangsdaten überlassen, damit er spezielle Funktionen des Accounts verwenden kann. Die erste Funktion des Schriftformerfordernisses erfüllen Zugangsdaten im Internet somit nicht.

348 Die zweite Funktion des Schriftformerfordernisses besteht darin, Fälschungen zu erschweren ${ }^{153}$ Manche Stimmen der Literatur behaupten, dass bei Accounts im Internet, die eine rein wissensbasierte Authentisierung verwenden, das Fälschungsrisiko erheblich geringer sei als bei Vollmachtsurkunden, weil das Ausspähen des Passworts viel schwieriger sei ${ }^{154}$ Aus zwei Gründen kann dem nicht zugestimmt werden. Zum einen kann eine Unterschrift zwar recht leicht nachgemacht werden. Die Handschrift ist jedoch ein bei jeder Person unterschiedliches Sein-Merkmal ${ }^{155}$ sodass es sehr schwer ist, diese nachzumachen, ohne dass die Fälschung aufgedeckt werden kann. Zugangsdaten hingegen sind ein Geheimnis, sodass ein Dritter sie mittels vielfältiger Möglichkeiten ${ }^{156}$ in Erfahrung bringen kann. Kennt

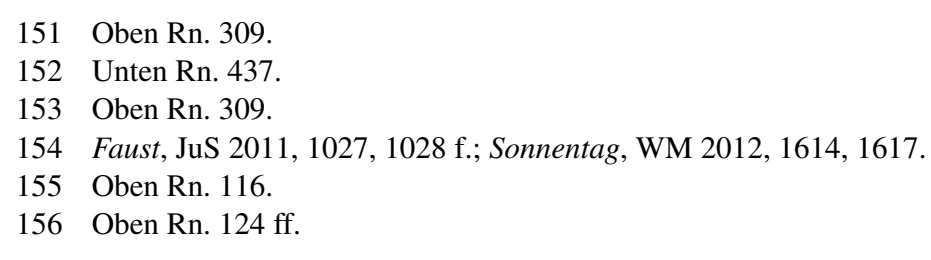


der Angreifer die Zugangsdaten, kann er sie einsetzen, wie der AccountInhaber. Für einen Dritten ist dann im Gegensatz zur Unterschrift nicht überprüfbar, dass ein Dritter gehandelt hat. Bei gefälschten Unterschriften gibt es keine Rechtsscheinhaftung, sodass diese auch nicht auf Zugangsdaten im Internet übertragen werden kann ${ }^{157}$ Gegen die Behauptung, das Fälschungsrisiko bei Accounts ohne Überprüfung der Identität sei geringer als bei Vollmachtsurkunden, spricht, dass diese Accounts durch einen Dritten erstellt werden können 158 Die Echtheit eines Accounts lässt sich im Nachhinein im Gegensatz zur Unterschrift nicht überprüfen ${ }^{159}$ Insofern muss ein Angreifer noch nicht einmal Zugangsdaten vom Account-Inhaber ausspähen. Die zweite Funktion des Schriftformerfordernisses, eine Fälschung zu erschweren, ist ebenfalls nicht erfüllt. Ferner ist durch die fehlende Unterschrift die Rückkopplung an den Geschäftsherren abgeschwächt. Der Geschäftsherr hat bei dem Blankett durch seine Unterschrift teilweise eine Erklärung geschaffen ${ }^{160}$ Mit der Erstellung seines Accounts hat der Account-Inhaber im Vergleich dazu jedoch nur die Möglichkeit geschaffenen, neue Erklärungen zu abzugeben. Im Vergleich zum Blankett stellt sich dies nur als Unterschriftsmöglichkeit dar ${ }^{161}$ Der Account-Inhaber gibt durch das Erstellen des Accounts noch keinen Inhalt für ein oder mehrere über den Account abschließbare Rechtsgeschäfte vor. Beim Missbrauch von Zugangsdaten im Internet von Accounts, die eine rein wissensbasierte Authentisierungsmethode einsetzen, sind die zentralen Aspekte der Stärke des Rechtsscheintatbestandes des $\S 172$ Abs. 1 BGB nicht gegeben.

Selbst die Voraussetzung, dass der Umfang der Vollmacht benannt ist, wodurch Missbrauchsmöglichkeiten eingeschränkt werden, ist beim Missbrauch von Zugangsdaten im Internet nicht in vergleichbarer Weise vorhanden. Bei der Vollmachtsurkunde wird durch die Angabe des Umfangs sichergestellt, dass einem möglichen Missbrauch durch Vertreter Grenzen gesetzt sind 162 Bei der Vollmachtsurkunde ist ein Missbrauch somit jedoch nur im begrenzten Umfang möglich. Die offene und verdeckte Blanketterklärung zeigen, dass dieses Erfordernis auch in vergleichbarer Weise durch eine andere Gestaltung erfolgen kann. Beim offenen und verdeckten

\footnotetext{
157 J. Münch, NJW-CoR 4/1989, 7, 9.

158 Oben Rn. 210

159 Unten Rn. 603

160 Wiebe, Elektronische Willenserklärung, S. 434.

161 Ebd. S. 434.

162 Oben Rn. 311
} 
Blankett ist zwar der Umfang der Ausfüllungsmacht in der Urkunde nicht benannt, sodass ein Missbrauch in größerem Umfang möglich ist. Weil ein Blankett im Original jedoch nur einmal ausgefüllt werden kann, ist der Missbrauch nur einmalig möglich. Eine im Umfang beschränkte, mehrfach nutzbare Missbrauchsmöglichkeit ist dabei mit einer einmaligen, umfänglicheren Möglichkeit vergleichbar. Bei den Zugangsdaten im Internet ist noch nicht einmal diese in unterschiedlichen Ausprägungen mögliche Voraussetzung der Beschränkung des Missbrauchs erfüllt. Hat ein Dritter die Zugangsdaten zu einem Account im Internet, kann er die gleichen Handlungen vornehmen, wie der Account-Inhaber. Beim Blankett ist durch die Anforderung, dass die Urkunde im Kern geschaffen sein muss, ein konkretes Rechtsgeschäft im noch zu bestimmenden Umfang vorgegeben. Ein Dritter kann mit dem Account des Inhabers jedoch eine Vielzahl von Rechtsgeschäften im gleichen Umfang, wie es der Account-Inhaber könnte, vornehmen. Die Voraussetzung, dass Missbrauchsmöglichkeiten durch den Rechtsscheintatbestand eingeschränkt werden, ist somit ebenfalls nicht gegeben.

350 Bei der Vollmachtsurkunde sowie beim Blankett liegt ein eigenes Handeln des Geschäftsherren vor, das rechtsgeschäftlichen Charakter hat. Ohne diesen rechtsgeschäftlichen Charakter geht von der Urkunde kein ausreichender Rechtsschein aus ${ }^{163}$ Sowohl bei Vollmachtsurkunden als auch bei Blanketten gibt der Aussteller zumindest eine grobe Richtung des Rechtsgeschäfts vor. Bei der Vollmachtsurkunde schafft er eine vollständige eigene Willenserklärung mit rechtsgeschäftlichem Charakter. Bei der Blanketturkunde erstellt er zumindest einen Teil davon. Bei der Weitergabe von $\mathrm{Zu}$ gangsdaten im Internet fehlt dieser rechtsgeschäftliche Charakter des Handelns des Account-Inhabers. Er gibt weder eine Richtung für ein mögliches Rechtsgeschäft vor, noch tritt sein Verhalten nach außen, sodass der Rechtsverkehr ein schützenswertes Vertrauen aufgrund seiner Handlung entwickeln könnte.

351 Die zentralen und nebensächlichen Anforderungen an die Stärke des Rechtsscheintatbestandes des $\S 172$ Abs. 1 BGB sind somit beim Missbrauch von Zugangsdaten im Internet von Accounts, die eine rein wissensbasierte Authentisierung einsetzen, nicht gegeben. Nachfolgend sollen noch einige Argumente, die für eine Anerkennung eines Rechtsscheintatbestandes vorgebracht werden, relativiert oder widerlegt werden. Zunächst wird für den Rechtsscheintatbestand angeführt, dass die Missbrauchsmöglich-

163 Canaris, Vertrauenshaftung, S. 61, 443. 
keiten der Anerkennung nicht schaden ${ }^{164}$ Eine Rechtsscheinhaftung für abredewidrig ausgefüllte Blanketterklärungen wäre nicht begründbar, weil bei einer teilweise offen gelassenen Willenserklärung die Missbrauchsmöglichkeiten hoch sind ${ }^{165}$ Ganz im Gegenteil wird die Haftung gerade mit der Gefährlichkeit der Blanketterklärung und deren Anfälligkeit für Missbrauchsmöglichkeit begründet ${ }^{166}$ Die Missbrauchsmöglichkeiten sprechen somit zwar nicht grundsätzlich gegen die Anerkennung einer Haftung, sagen jedoch nichts zur Vergleichbarkeit der Stärke des Rechtsscheintatbestandes aus.

Bei der Vollmachtsurkunde wird die Vertretungskonstellation ebenso wie beim Ausfüllen des offenen Blanketts vor den Augen des Geschäftsgegners deutlich. Zugangsdaten im Internet erfüllen zugleich Identifikations- und Legitimationsfunktion ${ }^{167}$ Bei ihrem Missbrauch kann der Erklärungsempfänger nicht erkennen, dass ein Dritter gehandelt hat. Insofern kann er kein schützenswertes Vertrauen in eine möglicherweise vorhandene Vollmacht des Dritten entwickeln, wie er es bei Vollmachtsurkunde und offenem Blankett kann. Die analoge Anwendung auf die verdeckte Blanketterklärung zeigt jedoch, dass auf die Erkennbarkeit der Drei-Personen-Konstellation verzichtet werden kann 168

Einzelne Stimmen der Literatur erwägen, dass der Geschäftsgegner schutzwürdiger sei, weil er das Handeln des Dritten nicht erkennen könne ${ }^{169}$ Zwar kann der Erklärungsempfänger der Erklärung nicht ansehen, ob der Account-Inhaber oder ein Dritter sie abgegeben hat. Dies ist jedoch nur eine Zustandsbeschreibung der Kommunikationsmodalitäten. Eine Schutzwürdigkeit begründet dies nicht. Der Erklärungsempfänger möchte ebenso wie der Account-Inhaber von den Vorteilen der schnellen Kommunikation profitieren, sodass er die Nachteile gleichfalls mit in Kauf nimmt. Ob er deswegen schutzwürdig ist, kann sich nicht aus der Zustandsbeschreibung, sondern nur aus einer angemessenen Risikoverteilung ergeben. Die entscheidende Frage ist, mit welcher Sicherheit sich der Erklärungsempfän-

164 Oechsler, AcP 208 (2008), 565, 579 sowie implizit auch Bork ${ }^{3}$, Rn. 1411.

165 Oechsler, AcP 208 (2008), 565, 579.

$166 B G H$, Urteil v. 25. 11. 1963, II ZR 54/61 - BGHZ 40, 297, 305: „,dass es allein darauf ankommt, wie der redliche Dritte, der den Blankettmißbrauch nicht kannte, die Erklärung [... ] auffassen durfte“.

167 Oben Rn. 120

168 Vgl. oben Rn. 337

169 Faust, BGB AT ${ }^{3}$, 26 Rn. 41; ders., JuS 2011, 1027, 1028. 
ger aufgrund der objektiv erkennbaren Tatsachen auf das Vorliegen einer Legitimation verlassen kann, also die Stärke des Rechtsscheintatbestandes.

354 Die angemessene Risikoverteilung solle jedoch eine analoge Anwendung des $\S 172$ Abs. 1 BGB rechtfertigen $\left[{ }^{170}\right.$ Beide Seiten wählen das Medium inklusive der dazugehörigen Risiken. Verbraucher werden gesetzlich häufig von Risiken befreit. Von der Zurechnung einer Willenserklärung zum Kauf beim Fernabsatz kann sich ein Verbraucher, der bei einem Unternehmen kauft, ohnehin nach § 312d Abs. 1 S. 1 BGB lösen. Im Verkehr zwischen zwei Kaufleuten oder zwei Nicht-Kaufleuten untereinander hingegen sei eine einseitige Risikoauferlegung unangemessen ${ }^{171}$ Ohne Anerkennung einer Rechtsscheinhaftung wären Schutzbehauptungen Tür und Tor geöffnet ${ }^{172}$ Diese Situation des ,Widerrufsrecht kraft Beweislast“ solle verhindert werden ${ }^{173}$ Dies begründet jedoch weder, warum Schutzbehauptungen zu verhindern sind, noch ob die Risikoverteilung anschließend angemessen ist 174

355 Zusammenfassend lässt sich festhalten, dass die Umstände, die den Rechtsschein bei Vollmachtsurkunden sowie Blanketten begründen, beim Missbrauch von Zugangsdaten im Internet bei einer rein wissensbasierten Authentisierung nicht vorhanden sind. Ein Rechtsscheintatbestand, der so stark ist, dass er eine analoge Anwendung des § 172 Abs. 1 BGB rechtfertigt, besteht somit insoweit nicht. Bei der noch zu untersuchenden ZweiFaktor-Authentisierung kann sich ein abweichendes Ergebnis ergeben ${ }^{175}$

\section{bb) Zurechenbarkeit}

356 Es stellt sich die Frage, ob hinsichtlich der Zurechenbarkeit zwischen der Vollmachtsurkunde und den Zugangsdaten im Internet eine vergleichbare Interessenlage besteht. Dazu sollen die beiden Komponenten der Zurechenbarkeit beleuchtet werden. Der Rechtsschein muss dem Account-Inhaber durch dessen Handeln subjektiv zurechenbar sein und er muss objektiv die Möglichkeit haben, den Rechtsschein zu verhindern.

170 Oechsler, AcP 208 (2008), 565, 579.

171 Ebd. 579.

172 Ebd. 579.

173 Mankowski, CR 2003, 44; ders., MMR 2004, 181.

174 Dazu ausführlich unten Rn.625ff.

175 Siehe unten Rn. 578 ff. 
Bei der subjektiven Zurechnung lässt sich die vergleichbare Interessenlage mit dem Missbrauch von Zugangsdaten im Internet begründen. Bei der Vollmachtsurkunde sowie den Blanketten muss der Geschäftsherr dem Dritten die Urkunde aushändigen. Dies entspricht der Weitergabe der Zugangsdaten durch den Account-Inhaber. Nach dem Risikoprinzip ${ }^{176}$ stammt die willentliche Schaffung eines Rechtsscheintatbestandes aus der Sphäre des Geschäftsherren, sodass diese zurechenbar ist. Nach dem Verschuldensprinzip ${ }^{177}$ stellt dies eine vorsätzliche Schaffung des Rechtsscheintatbestandes dar, die zurechenbar ist.

Vereinzelt wird einschränkend vertreten, dass die Zurechenbarkeit nur begründet ist, wenn der Account-Inhaber wenigstens Eventualvorsatz bezüglich des späteren Missbrauchs durch den Handelnden hatte ${ }^{178}$ Begründet wird diese Einschränkung mit dem Erfordernis des wissentlichen Schaffens eines Rechtsscheintatbestandes, wie es in $\S \S 170$ ff. BGB gefordert wird. Der Geschäftsherr verursache den „Rechtsscheintatbestand (abredewidrig ausgefülltes Blankett) nicht in positiver Kenntnis. 4179 Diese Begründung verfehlt die relevanten Aspekte der Zurechnung des Rechtsscheins. Die gesetzlichen Rechtsscheintatbestände zeigen, dass nur der Rechtsschein willentlich gesetzt werden muss, nicht jedoch die eventuell resultierende Haftung vom Vorsatz umfasst sein muss. Nach $§ 172$ Abs. 1 BGB ist ein willentliches Aushändigen erforderlich. Der spätere Missbrauch der Vertretungsmacht ist kein Erfordernis des $\$ 172$ Abs. 1 BGB. Der systematische Blick auf den gutgläubigen Erwerb von beweglichen Sachen ( $\S 932$ ff. BGB) bestätigt dies. Der Rechtsschein des Besitzes ( $\$ 1006$ Abs. 1 BGB) ist zurechenbar, wenn der Eigentümer den Besitz der Sache einem anderen verschafft hat (vgl. § 935 Abs. 1 BGB). Ein voluntatives Element, dass der Eigentümer schon bei der Übertragung des unmittelbaren Besitzes mit einer späteren Veräußerung durch den neuen Besitzer rechnet, ist hingegen nicht erforderlich. Das einzig relevante Moment bei der Zurechnung des Rechtsscheins beim Missbrauch nach Weitergabe der Zugangsdaten ist daher, dass der Account-Inhaber dem Handelnden ermöglicht, sich als Account-Inhaber zu gerieren. Die Einschränkung, dass der Account-Inhaber Eventualvorsatz

\section{Dazu oben Rn. 243}

177 Dazu oben Rn. 237

178 Schnell, S. 267. Für das abredewidrig ausgefüllte Blankett: Gerd Müller, AcP 181 (1981), 515, 534.

179 Gerd Müller, AcP 181 (1981), 515, 534. Schnell, S. 267 begründet die Einschränkung nicht. 
bezüglich eines späteren Missbrauchs haben muss, kann daher nur gerechtfertigt werden, wenn neben dem willentlichen Schaffen des Rechtsscheintatbestandes nach dem Verschuldensprinzip ${ }^{180}$ auch die fahrlässige Schaffung des Rechtsscheintatbestandes ausreicht.

359 Ferner lässt sich ein Argument, das zur Begründung der Anscheinsvollmacht verwendet wird, fruchtbar machen. Bei der Anscheinsvollmacht ist der Rechtsschein zurechenbar, wenn jemandem eine Stellung eingeräumt wird, die typischerweise mit einer Vertretungsmacht verbunden ist ${ }^{181}$ Die Überlassung der Zugangsdaten ist typischerweise mit einer Vertretungsmacht verbunden. Eine Handlung über den Account könnte daher den Rechtsschein erwecken, der Account-Inhaber habe sich rechtsgeschäftlich verpflichtet.

360 Die willentliche Mitteilung der Zugangsdaten kann daher die subjektive Komponente der Zurechnung begründen. Dabei ist jedoch fraglich, was unter einer willentlichen Weitergabe zu verstehen ist. Eine explizite Mitteilung der Zugangsdaten ist jedenfalls erfasst. Problematisch ist hingegen, was unter der Weitergabe noch zu verstehen ist. Bei einem weiten Verständnis des Begriffs der Weitergabe ${ }^{182}$ fällt auch die Kenntnisnahme des Dritten durch das Lesen einer Notiz der Zugangsdaten ${ }^{183}$ oder das Speichern in der Schlüsselbund-Verwaltung ${ }^{184}$ darunter. Bei dem hier zugrunde gelegtem engen Verständnis der Weitergabe ${ }^{185}$ fällt nur die Weitergabe des AccountInhabers an einen Dritten im Bewusstsein, dass dieser die Zugangsdaten später eigenständig nutzen wird, darunter. Offenbart der Account-Inhaber einem Angreifer im Rahmen eines Phishing-Angriff ${ }^{186}$ durch Täuschung die Zugangsdaten, liegt keine Weitergabe vor.

361 Eine vergleichbare Interessenlage zu $\S 172$ Abs. 1 BGB besteht beim Missbrauch von Zugangsdaten im Internet dann, wenn der Account-Inhaber die Zugangsdaten weitergibt. Daraus lässt sich schließen, dass ein etwaiger Rechtsscheintatbestand bei Weitergabe zurechenbar ist. Davon getrennt ist die Frage, ob auch ohne Weitergabe der Zugangsdaten, ein Rechtsscheintat-

180 Dazu oben Rn.237 ff.

181 Schramm, in: MüKo-BGB $^{6}, \S 167$ Rn. 62.

182 So Borges, Elektronischer Identitätsnachweis, S. 136; ders., NJW 2011, 2400, 2403; Sonnentag, WM 2012, 1614, 1618; Verse/Gaschler, Jura 2009, 213, 215 f.

183 Dazu oben Rn. 132

184 Dazu oben Rn. 135

185 Oben Rn. 295

186 Oben Rn. $138 \mathrm{ff}$. 
bestand zurechenbar ist ${ }^{187}$ Die analoge Anwendung des $\S 172$ Abs. 1 BGB auf das fahrlässige Abhandenkommen der Urkunde kann dafür eine maßgebliche Wertung geben. Die Frage, ob ohne Weitergabe der Zugangsdaten eine Zurechnung in Betracht kommt, kann in die gleiche Richtung entschieden werden, ob das fahrlässige Ermöglichen des Abhandenkommens der Vollmachtsurkunde ausreicht ${ }^{188}$ Bei der objektiven Komponente der Zurechnung besteht somit eine vergleichbare Interessenlage.

Fraglich ist, ob auch bei der objektiven Komponente eine vergleichbare Interessenlage besteht. Objektiv muss derjenige, der den Rechtsschein geschaffen hat, die Möglichkeit haben diesen zu verhindern oder zu zerstören ${ }^{189}$ Bei der Vollmachtsurkunde ist die objektive Zurechnung in $§ 172$ Abs. 2 BGB geregelt. Der Rechtsschein der Vollmachtsurkunde besteht solange, bis diese zurückgegeben ist oder für kraftlos erklärt wird. Der Geschäftsherr hat somit zum einen die Möglichkeit den Rechtsscheinträger, den Besitz an der Urkunde, wieder an sich zu nehmen. Zum anderen kann er, wenn dies scheitern sollte, die Urkunde für kraftlos erklären, sodass er in jedem Fall eine Möglichkeit hat, den Rechtsscheintatbestand zu zerstören.

Diese Möglichkeiten hat der Account-Inhaber bei den Zugangsdaten nicht in gleichem Maße. Bei einer rein wissensbasierten Authentisierung erfolgt bei einer Weitergabe die Duplizierung des Wissens um die Zugangsdaten. Das Wissen um die Zugangsdaten ist nicht wie die Erklärung bei der Vollmachts- oder Blanketturkunde in einer Besitz-Komponente perpetuiert ${ }^{190}$ Das Wissen um die Zugangsdaten kann sich der Account-Inhaber nicht zurückholen. Es ist jedoch zu erwägen, ob die Möglichkeit, das Kennwort zu ändern, eine vergleichbare Methode ist, den Rechtsschein zu zerstören. Solange der Dritte, der das Passwort kennt, dieses nicht ändert, kann der Account-Inhaber den Zugang des Dritten zum Account unterbinden. Dazu hat der Account-Inhaber aber erst Anlass, wenn er von dem ersten Missbrauch erfährt. Ändert der Dritte das Kennwort und womöglich auch die E-Mail-Adresse, über die ein vergessenes Kennwort geändert werden kann, so hat der Account-Inhaber nur schwer die Möglichkeit den Rechtsschein zu unterbinden ${ }^{191}$ Dies stellt einen großen Unterschied zur Interessenlage bei der Vollmachtsurkunde dar. Der Geschäftsherr kann, falls

187 Dazu unten Rn.671 ff.

188 Dazu oben Rn. 315

189 Oben Rn. 246

190 Zur Bedeutung der Perpetuierungsfunktion Oechsler, AcP 208 (2008), 565, 577.

191 J. Hoffmann, in: Leible/Sosnitza, Rn. 177. 
er den Besitz der Vollmachtsurkunde vom Vertreter nicht zurückerlangen kann, den Rechtsschein durch Kraftloserklärung verhindern. Eine vergleichbare Interessenlage zwischen $§ 172$ Abs. 1 BGB und dem Missbrauch von Zugangsdaten im Internet besteht somit bezüglich der objektiven Zurechnungskomponente nicht.

\section{f) Zwischenergebnis}

364 Eine analoge Anwendung des $\S 172$ Abs. 1 BGB auf den Missbrauch von Zugangsdaten im Internet kommt somit bezüglich Accounts, die auf eine rein wissensbasierte Authentisierung setzen, nicht in Betracht.

\section{Zwischenergebnis}

365 Die Haftung für den Missbrauch von Zugangsdaten im Internet kann nach deren Weitergabe daher nicht überzeugend durch eine analoge Anwendung des $\S 172$ Abs. 1 BGB begründet werden, da deren Voraussetzungen nicht vorliegen. Eine vergleichbare Interessenlage bezüglich des Rechtsscheintatbestandes besteht nicht. Zwar zeigt die analoge Anwendung auf verdeckte Blanketterklärungen, dass auf die Erkennbarkeit des Handelnden verzichtet werden kann 192 Die wesentlichen Elemente des Rechtsscheins der Vollmachtsurkunde sind jedoch bei Zugangsdaten im Internet nicht gegeben 193 Werden lediglich die Wertungen der Zurechenbarkeit übertragen, entsteht dadurch eine Rechtsscheinhaftung ohne Rechtsscheintatbestand ${ }^{194}$ Bei der Zurechenbarkeit besteht zwar im Hinblick auf das Handeln des Account-Inhabers eine vergleichbare Interessenlage ${ }^{195}$ Mangels vergleichbarer Möglichkeiten den Rechtsschein zu zerstören, ist nicht einmal bezüglich der $\mathrm{Zu}$ rechenbarkeit eine vergleichbare Interessenlage vorhanden 196

\section{Oben Rn. 352}

193 Oben Rn. 345 ff.

194 Dies wird ebenfalls der Lösung über die Anscheinsvollmacht vorgeworfen, unten Rn. 380

195 Oben Rn. 357

196 Oben Rn. 362 


\section{Zwischenergebnis}

Die Haftung für Missbrauch von Zugangsdaten im Internet kann weder durch die Duldungsvollmacht noch durch analoge Anwendung des § 172 Abs. 1 BGB überzeugend gelöst werden. Die Duldungsvollmacht passt strukturell nicht auf die Konstellation der Zugangsdaten im Internet, weil das Handeln des Dritten nicht erkennbar wird 197 Die analoge Anwendung des $§ 172$ Abs. 1 BGB scheitert an einer Vergleichbarkeit der Stärke der Rechtsscheintatbestände 198

Bei Weitergabe der Zugangsdaten besteht eine im Ergebnis bisher unbestrittene Meinung, dass der Account-Inhaber auch bei einer rein wissensbasierten Authentisierungsmethode für den Missbrauch der Zugangsdaten hafte. Diese Meinung steht - wie noch zu zeigen sein wird - im Widerspruch zu den Ansichten bei Konstellationen ohne Weitergabe der Zugangsdaten 199 Im Laufe der weiteren Untersuchung wird gezeigt, dass man die Haftung für den Missbrauch von Zugangsdaten sowohl mit und ohne deren Weitergabe überzeugend über die allgemeine Rechtsscheinhaftung lösen kann 200

Der Geschäftsgegner des Account-Inhabers ist selbst, wenn eine Rechtsscheinhaftung scheitert, nicht schutzlos gestellt. Insbesondere in Zwei-Personen-Konstellationen, in denen eine vertragliche Vereinbarung zwischen dem Geschäftsgegner und dem Account-Inhaber besteht, dürften vertragliche Ansprüche regelmäßig einschlägig sein 201

197 Oben Rn. 302

198 Oben Rn. $344 \mathrm{ff}$.

199 Dazu ausführlich unten Rn. 667

200 Unten Rn. 489 ff.

201 Zur Lösung über die vertraglichen Beziehungen unten Rn. 397 
